\title{
Life-form spectra of quartzite and itabirite rocky outcrop sites, Minas Gerais, Brazil
}

\author{
Maria Cristina Teixeira Braga Messias ${ }^{1,4}$, Mariangela Garcia Praça Leite ${ }^{2}$, \\ João Augusto Alves Meira-Neto ${ }^{3}$ \& Alessandra Rodrigues Kozovits ${ }^{1}$ \\ ${ }^{1}$ Departamento de Biodiversidade, Evolução e Meio Ambiente, \\ Universidade Federal de Ouro Preto, CEP 35400-000, Ouro Preto, MG, Brazil \\ ${ }^{2}$ Departamento de Geologia, Universidade Federal de Ouro Preto, CEP 35400-000, Ouro Preto, MG, Brazil \\ ${ }^{3}$ Departamento de Biologia Vegetal, Universidade Federal de Viçosa, CEP 36570-000, Viçosa, MG, Brazil \\ ${ }^{4}$ Corresponding author: cristina@iceb.ufop.br
}

MESSIAS, M.C.T.B., LEITE, M.G.P., MEIRA-NETO, J.A.A., KOZOVITS, A.R. Life-form spectra of quartzite and itabirite rocky outcrop sites, Minas Gerais, Brazil. Biota Neotrop. 11(2): http://www.biotaneotropica.org. br/v11n2/en/abstract?article+bn01311022011

\begin{abstract}
Vascular species and their respective life-forms and coverage were recorded in a Brazilian quartzite and itabirite rocky outcrop site at Serra de Ouro Preto, producing the floristic, frequency and vegetational spectra. Three habitats in both lithologies were defined by geomorphology as: 1) Sloped areas near the mountain summit, with grasslands; 2) Plateaus in the middle of the slope, with grasslands; and 3) Lower and/or concave parts of the slopes, with woody savannas. The life-forms followed Raunkiaer's System. We aimed to answer the following questions: Do quartzite and itabirite rocky outcrops have different biological spectra? Are the biological spectra different in the geomorphologic habitats? Do the floristic, vegetational and frequency spectra differ from one another? What spectrum stacks up to a rocky outcrop physiognomy description? The results portrayed that: a) the most represented life-forms were the phanerophytes and hemicryptophytes; b) the floristic and frequency spectra did not differ from each other, but both differed from the vegetational one; c) all the floristic spectra were similar, but there were significant differences in the frequency and vegetational spectra among the lithology and geomorphology habitats; d) higher phanerophyte and lesser hemicryptophyte coverages were found in the itabirite areas and also in the lower or concave parts of the slopes of both lithologies; and e) the vegetational spectrum was more efficient for the studied rocky outcrop comparison. Relationships between the environmental aspects and life-form spectra are discussed. This study will help advance the development of restoration projects for these areas by adding knowledge of their flora composition, structure and function.
\end{abstract}

Keywords: biological spectrum, ferruginous rocky outcrops, Quadrilátero Ferrífero, Raunkiaer.

MESSIAS, M.C.T.B., LEITE, M.G.P., MEIRA-NETO, J.A.A., KOZOVITS, A.R. Formas de vida em campos rupestres sobre quartzito e itabirito, em Minas Gerais, Brasil. Biota Neotrop. 11(2): http://www. biotaneotropica.org.br/v11n2/pt/abstract?article+bn01311022011

Resumo: Realizou-se um levantamento das espécies vasculares e suas respectivas formas de vida e coberturas, em campos rupestres na Serra de Ouro Preto, sobre quartzito e itabirito, para construção dos espectros florísticos, de freqüência e vegetacional. Em cada litologia foram delimitados, pela geomofologia, três tipos de habitats: 1) Áreas inclinadas, nos topos das montanhas, com campos limpos; 2) Platôs, na parte mediana das encostas, com campos limpos e 3) Partes mais baixas ou côncavas das encostas, com campos sujos. As formas de vida seguiram o sistema de Raunkiaer. Averiguaram-se as seguintes questões: Campos rupestres sobre itabirito e quartzito possuem diferentes espectros biológicos? Os habitats estratificados pela geomorfologia diferem quanto aos espectros biológicos? Os espectros florísticos, de frequência e vegetacional diferem entre si? Qual espectro se destaca como descritor da fisionomia de campos rupestres? Os resultados evidenciaram que: a) fanerófitas e hemicriptófitas são as formas de vida predominantes; b) os espectros florísticos e de frequência não diferiram entre si e ambos foram estatisticamente diferentes do espectro vegetacional; c) os habitats estratificados pela litologia e geomorfologia apresentaram espectros florísticos similares, mas os espectros de frequência e vegetação mostraram diferenças significativas; d) maior cobertura de fanerófitas e menor cobertura de hemicriptófitas foram encontradas nas áreas sobre itabirito, assim como nas partes mais baixas ou côncavas das encostas, em ambas litologias; e e) o espectro vegetacional mostrou-se mais eficiente para comparação dos habitats. Aspectos do ambiente relacionados com a distribuição de formas de vida são discutidos. Este estudo subsidia programas de restauração ambiental, ampliando o conhecimento da vegetação de campos rupestres.

Palavras-chave: espectro biológico, campos ferruginosos, Quadrilátero Ferrífero, Raunkiaer. 


\section{Introduction}

Campos Rupestres are also known as montane savannas, a kind of Brazilian rocky outcrop, that mainly occurs in the Espinhaço Range (Cadeia do Espinhaço), which extends northward from the State of Minas Gerais, to the State of Bahia, between $20^{\circ} 35^{\prime} \mathrm{S}$ and $11^{\circ} 11^{\prime} \mathrm{S}$ (Meguro et al. 1994). They are located in contact zones between the Cerrado (the main Neotropical savanna) and the Atlantic Forest (southern Espinhaço), as well as in transition zones of the Caatinga, the Cerrado and the Atlantic Forest (central and northern Espinhaço), important terrestrial hotspots. They harbor a great biodiversity and many endemic and threatened species (Giulietti \& Pirani 1988). The dominant vegetation is herbaceous and presents a mosaic of shrubs and subshrubs growing in a wide variety of substrates, including rocks and recently-decomposed stony, sandy or colluvial soils. The most common type of rock in this region is quartzite (Benites et al. 2007). According to Harley \& Simmons (1986), "campos rupestres" is the expression that defines the vegetation growing on quartzite-sandstone substrate, although this terminology is used to refer to Brazilian vegetation found on other kinds of rocky substrate such as granitic-gneiss (e.g. Queiroz et al. 1996) or itabirite (e.g. Jacobi \& Carmo 2008, Mourão \& Stehmann 2007, Viana \& Lombardi 2007). In the Quadrilátero Ferrifero, Southeastern Brazil, rocky outcrops occur frequently in areas with quartzite rocks, interspersed with itabirite, metasandstone, phyllite, schist and gneiss. Itabirite is frequently covered with a layer of duricrusts, formed by the rock's lateritic chemical weathering, known as cangas (Rosière \& Chemale Junior 2000)

Rocky outcrop plant communities are basically controlled by edaphic conditions, and often represent islands of xeric communities rising in a matrix of mesophytic vegetation (Porembski et al. 1994, 1998). Species show adaptation to over-heating or drought, such as: trichomes or persistent leaf sheaths for isolation and water uptake and accumulation, succulence, sclerophylly and desiccation tolerance, in the so-called resurrection plants (Gaff 1977, 1987, Porembski \& Barthlott 2000). The vegetation of ironstone outcrops also exhibits adaptations to living in a substrate rich in heavy metals like Iron, Manganese, Copper, Lead and others (Vincent \& Meguro 2008). Little is known about the communities in iron-rich rocks compared to other kinds of rocky outcrops (Jacobi et al. 2007, Jacobi \& Carmo 2008, Mourão \& Stehmann 2007, Silva 1991, Viana \& Lombardi 2007, Vincent 2004, Vincent \& Meguro 2008). In Brazil, plant communities in itabirite rocks occur in two main areas: Serra dos Carajás, in the Amazon region and Quadrilátero Ferrífero in the Southeast. These areas are threatened mainly by the high intensity of open pit mining.

According to Cain (1950), life-form studies are almost as important as the floristic composition in vegetation description. Plants can be categorized into different life-form classes depending on their similarities in structure and function (Mueller-Dombois \& Ellenberg 1974, Harrison et al. 2010). A life-form is characterized by the plant's adaptations to specific ecological conditions (Mera et al. 1999). Raunkiaer (1934) suggested a system for the classification of the life-forms of plants based on the position and degree of protection of the renewing buds, which are responsible for the renewal of the plant's aerial body on arrival of the season favorable to growth. In Raunkiaer's (1934) system, the harsher the unfavorable season is, the more protected the renewing buds are. This author stated five major classes, ordered according to increased protection of the renewing buds: phanerophytes, chamaephytes, hemicryptophytes, cryptophytes and therophytes. The original classification of Raunkiaer was changed by Mueller-Dombois and Ellenberg (1974), among others, to add plant features in the favorable season. Raunkiaer's system was strongly criticized (Sarmiento \& Monasterio 1983), but is still considered, in several ways, the most fundamental explanation for why certain combinations of plant species co-occur in a given habitat (Begon et al. 1996, Lomolino et al. 2005).

A floristic spectrum is created with a species list, where every species has the same weight. However, the usefulness of life-form spectra, for ecological investigations, seems to depend largely on some means of evaluating the species composing the flora of the communities (Cain 1950). The weighting of the species could be accomplished by the use of any quantitative data concerning the species, such as number of individuals, frequency or coverage. When the number of individuals of each life-form is counted, instead of species, each class can be weighted by its abundance, resulting in the vegetation's biological spectrum. This feature describes the vegetation rather than the flora, and allows comparisons to other spectra similarly constructed for other sites. However, Raunkiaer (1934) asserted that counting plant individuals in a survey is complicated, because sometimes it is difficult to distinguish what an individual is. For this reason, this author proposed a frequency spectrum, using the number of sampling units in which the species is present to weight the species.

In a Brazilian granitic rocky outcrop, Caiafa \& Silva (2005) found that the floristic spectra were different in the different habitats, where the grasslands had a higher percentage of hemicryptophytes followed by chamaephytes; and the scrub boundaries are more phanerophytic. Conceição \& Giulietti (2002), Conceição \& Pirani (2005) and Conceição et al. (2007a, b), working in quartzite-sandstone rocky outcrop areas, described these communities as having a high percentage of hemicryptophytes and a lack of therophytes. Little is known about life form spectra in ferruginous rocky outcrops (Jacobi et al. 2007).

There are few quantitative studies in ferruginous rocky outcrops in relation to quartzitic and granitic ones; known only are those of Jacobi et al. (2007), Jacobi \& Carmo (2008), Vincent (2004) and Vincent \& Meguro (2008). The accomplishment of ecological studies in Brazilian rock outcrops is important to improve the knowledge of Brazilian phytogeography by understanding the adaptation of plants to the environment and how they contribute to vegetational structure and physiognomy. Since the ferruginous rocky outcrops are highly threatened by mining activities, the ecological knowledge of this environment will also help advance the development of restoration projects for these areas.

The aim of this study was to answer the following questions: Do the floristic, vegetational and frequency spectra differ from quartzite to itabirite areas? Are the biological spectra different in the different geomorphologic habitats? What classes characterize these rocky outcrop life-form spectra? Do the floristic, vegetational and frequency spectra differ from one another? What aspects of the environment might be related with the distributions of life-form? What spectrum stacks up to a rocky outcrop physiognomy description?

\section{Material and Methods}

\section{Study sites}

This study was carried out in the Municipalities of Ouro Preto and Mariana, Minas Gerais State, southeastern Brazil, at about $20^{\circ}$ $10^{\prime} \mathrm{S}$ and $43^{\circ} 30^{\prime} \mathrm{W}, 890$ to 1250 meters above sea level. This area belongs to Samarco Mineração S.A. and is part of the Serra de Ouro Preto, South of Espinhaço Range. The climate according to Köppen is Cwb, i.e. mesothermic, with a rainy summer concentrated during November-March and a dry winter (Nimer 1989). The annual mean rainfall is $1250 \mathrm{~mm}$ and the annual mean temperature is around $20{ }^{\circ} \mathrm{C}$. Two campos rupestres areas, $12 \mathrm{~km}$ apart, with different 
kinds of rock (itabirite and quartzite), were selected to set the plots. The rocky outcrops in each lithology were stratified in three habitats by geomorphology as: 1) Sloped areas with bare rocks, near the mountain summit, with grasslands (campos limpos); 2) Plateaus, in the middle of the slope, with no more than $10 \%$ slope inclination, with grasslands; 3) Lower and/or concave parts of the slopes, in valleys or depressions, with woody savannas (campos sujos). Altogether six habitats were studied, determined by the two lithologies and three different geomorphologies. The grasslands in the sloped areas, established on itabirite (ISG), showed bare rocks with typical banded iron formation feature. The grasslands on the plateaus (IPG) had duricrusts formed by the lateritic chemical weathering of itabirite, known locally as cangas couraçadas. The woody savannas on itabirite (IWS) also had cangas partially covered by colluvial soil. On the other hand, the sloped grassland areas on quartzite (QSG) had a great amount of smooth bare rocks, and either shallow soil occupying the gaps between them, or forming small pools in their depressions. The grassland plateaus in the quartzitic areas (QPG) were comprised of a sandy, shallow and continuous soil with almost no bare rocks. The soil in this habitat showed poor drainage in the rainy season. The woody savannas on quartzite (QWS) presented sandy and colluvial soil with occasional bare rocks.

\section{Methods}

The survey was carried out from September/2007 to February/2009 by weekly field trips. In each lithology, thirty $10 \times 10 \mathrm{~m}$ plots, ten in each geomorphological habitat, were defined. All the vascular species were collected, identified and classified into life-forms according to Raunkiaer's System (Raunkiaer 1934). The cover area (\%) of each species was estimated as a measure of dominance (Mueller-Dombois \& Ellenberg 1974), by the vertical projection of the aerial parts of each species as a percentage of the total area of the plot (Westhoff \& Maarel 1978). The cover area estimation was assessed similar to the BraunBlanquet method (Mueller-Dombois \& Ellenberg 1974). The difference was that the cover area was evaluated as a continuous variable instead of using the categorical values of the Braun-Blanquet Cover-abundance Scale. After all species were recorded, the cover-abundance rating of each species was assigned from a point that had the best overview of the plot's entire plant assemblage. All the collected specimens were herborized and deposited in the Herbarium "Professor José Badini" (OUPR) of the Universidade Federal de Ouro Preto and grouped according to the Angiosperm Phylogeny Group (2009).

The data of each species life-form were used to construct the floristic, frequency and vegetational spectra (Raunkiaer 1934). Only Raunkiaer's major classes (phanerophytes, chamaephytes, hemicryptophytes, cryptophytes and therophytes) were considered in this analysis. The lianas and epiphytes were included in the phanerophyte class, while the geophytes and saprophytes were put in the cryptophyte class, as originally proposed by Raunkiaer (1934). In the frequency spectrum, each species was weighted by the number of plots in which it appeared, whereas in the vegetational spectrum, each life-form was weighted by its coverage (Mueller-Dombois \& Ellenberg 1974). In the construction of the biological spectra, each species was assigned to a single life-form class - the one in which the renewing buds were less protected. The biological spectra of each habitat were compared pairwise with a homogeneity analysis (Zar 1999) to test whether the life-form proportions were significantly different.

\section{Results}

In this survey, 263 vascular species were collected, belonging to 64 families, of which 135 (51.3\%) were considered phanerophytes; 88 $(33.5 \%)$, hemicryptophytes; $23(8.8 \%)$, chamaephytes; nine $(3.4 \%)$, cryptophytes and eight (3\%), therophytes. Among the phanerophytes, sensu Raunkiaer (1934), 13 species are lianas and one is epiphyte. The families with the greater number of species were Poaceae (28 species), Asteraceae (26), Melastomataceae (19), Fabaceae (17), Rubiaceae (14), Myrtaceae (13), Cyperaceae and Orchidaceae (12) (Table 1).

In the floristic, frequency and vegetational spectra (Figure 1), the most remarkable feature was the high percentage of phanerophytes. The floristic spectra from the different studied habitats did not present any significant difference (Table 2).

Significant differences were found among the frequency spectra of the different studied habitats (Table 2). When comparing the different habitats in the quartzite areas, there was not any significant difference among them, but there were differences when comparing them with the corresponding habitats in the itabirite areas. The frequency of phanerophytes is higher than the other life forms in every studied habitat, but in QSG and QPG the frequency of hemicryptophytes is closer to that of the phanerophytes (Figure 1). Furthermore, the highest frequency of chamaephytes was found in these two habitats. QSG showed a higher frequency of chamaephytes and a lower frequency of phanerophytes than in ISG. IWS presented a higher frequency of phanerophytes and cryptophytes and a lower frequency of chamaephytes, therophytes and hemicryptophytes in comparison to QWS. The frequency spectra did not present significant differences between the grassland (campos limpos) and woody savanna (campos sujos) physiognomies.

Significant differences among the vegetational spectra of the different studied habitats were found. (Table 2). Quartzite and itabirite

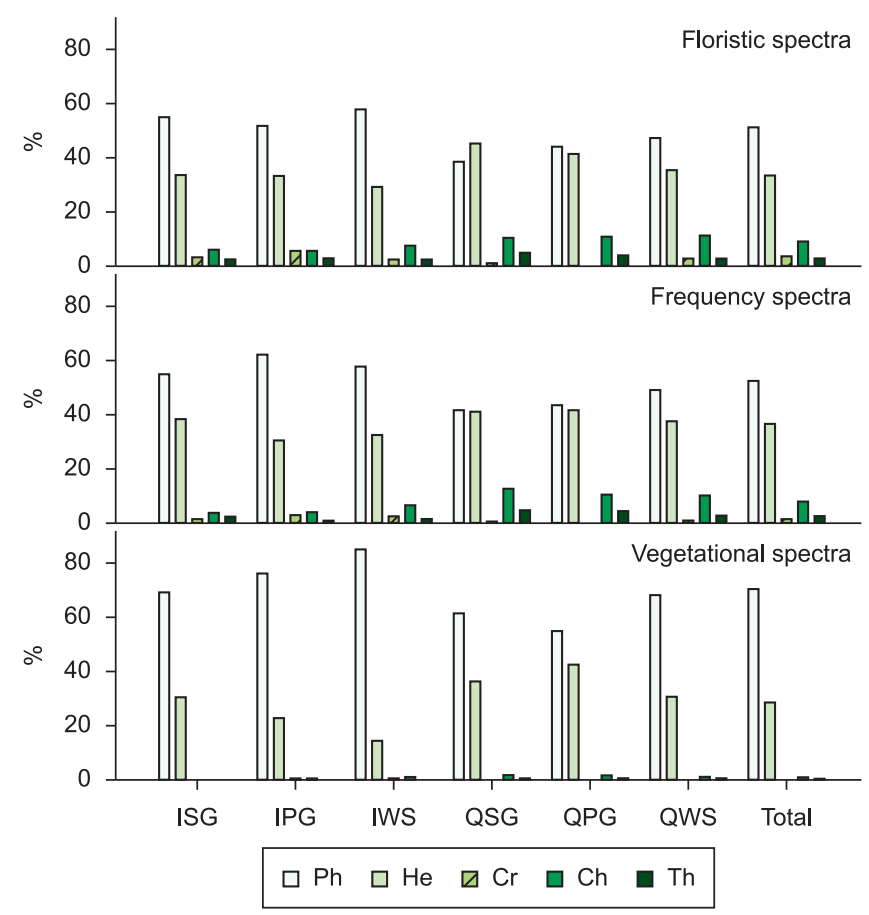

Figure 1. Floristic, frequency, and vegetational life-form spectra of rocky outcrop sites (approximately, $20^{\circ} 10^{\prime} \mathrm{S}-43^{\circ} 30^{\prime} \mathrm{W}$ ). $\mathrm{Ph}=$ phanerophyte; $\mathrm{He}=$ hemicryptophyte $\mathrm{Cr}=$ cryptophyte $\mathrm{Ch}=$ chamaephyte $\mathrm{Th}=$ therophyte; ISG $=$ Grasslands in sloped areas, with bare itabirite rocks, near the mountain summit; IPG = Grasslands on the itabirite plateaus, in the middle of the slope; IWS = Woody savannas in the lower and/or concave parts of the slopes, in valleys or depressions of the itabirite areas; $\mathrm{QSG}=$ Grasslands in sloped areas, with bare quartzite rocks, near the mountain summit; $\mathrm{QPG}=$ Grasslands on the quartzite plateaus, in the middle of the slope; QWS = Woody savannas in the lower and concave parts of the slopes, in valleys or depressions of the quartzite areas; Total $=$ All the six habitats. 
Table 1. Species list of rocky outcrop sites (approximately, $20^{\circ} 10^{\prime} \mathrm{S}-43^{\circ} 30^{\prime} \mathrm{W}$ ) and their respective life-form and mean coverage (\%) in the different habitats.

\begin{tabular}{|c|c|c|c|c|c|c|c|c|}
\hline \multirow[t]{2}{*}{ Family/Species } & \multirow[t]{2}{*}{ Voucher } & \multirow[t]{2}{*}{ LF } & \multicolumn{3}{|c|}{ Itabirite } & \multicolumn{3}{|c|}{ Quartzite } \\
\hline & & & ISG & IPG & IWS & QSG & QPG & QWS \\
\hline $\begin{array}{l}\text { Acanthaceae } \\
\quad \text { Ruellia macrantha (Mart. ex Ness) Lindau }\end{array}$ & 2115 & $\mathrm{Ph}$ & 0 & 0.03 & 0.18 & 0 & 0.02 & 0.31 \\
\hline $\begin{array}{l}\text { Anacardiaceae } \\
\quad \text { Tapirira guianensis Aubl. }\end{array}$ & 1898 & $\mathrm{Ph}$ & 0 & 0 & 0 & 0 & 1.42 & 3.82 \\
\hline $\begin{array}{l}\text { Anemiaceae } \\
\qquad \text { Anemia ferruginea Humb. \& Bonpl. ex Kunth }\end{array}$ & 2000 & $\mathrm{He}$ & 0 & 0 & 0 & 0.01 & 0 & 0 \\
\hline Anemia hirsuta (L.) Sw. & 1577 & $\mathrm{He}$ & 0 & 0 & 0.01 & 0 & 0 & 0 \\
\hline $\begin{array}{l}\text { Annonaceae } \\
\quad \text { Guatteria vilosissima A. St.-Hil. }\end{array}$ & 2268 & $\mathrm{Ph}$ & 0 & 0 & 0 & 0 & 0 & 0.35 \\
\hline Xylopia sericea A. St.-Hil. & 2066 & $\mathrm{Ph}$ & 0 & 0 & 0 & 0 & 0 & 0.7 \\
\hline $\begin{array}{l}\text { Apocynaceae } \\
\text { Blepharodon pictum (Vahl) W.D.Stevens }\end{array}$ & 1938 & $\mathrm{Li}$ & 0 & 0 & 0 & 0 & 0 & 0.02 \\
\hline Ditassa laevis Mart. & 1708 & $\mathrm{Li}$ & 0.01 & 0 & 0.02 & 0.01 & 0 & 0 \\
\hline Ditassa linearis Mart. & 1453 & $\mathrm{Li}$ & 0.03 & 0.04 & 0.01 & 0 & 0 & 0.02 \\
\hline Ditassa mucronata Mart. & 1797 & $\mathrm{Li}$ & 0.02 & 0.03 & 0.07 & 0 & 0.03 & 0.05 \\
\hline Forsteronia velloziana (A.DC.) Woodson & 2061 & $\mathrm{Li}$ & 0 & 0 & 0 & 0 & 0 & 0.02 \\
\hline Mandevilla tenuifolia (J.C.Mikan) Woodson & 1447 & Th & 0.05 & 0.01 & 0.01 & 0 & 0 & 0 \\
\hline Minaria decussata (Mart.) T.U.P.Konno \& Rapini & 1669 & $\mathrm{Ch}$ & 0 & 0.01 & 0 & 0 & 0 & 0 \\
\hline $\begin{array}{l}\text { Aquifoliaceae } \\
\quad \text { Ilex subcordata } \text { Reissek }\end{array}$ & 2069 & $\mathrm{Ph}$ & 0.44 & 0.33 & 0.82 & 0 & 0.08 & 0.08 \\
\hline $\begin{array}{l}\text { Araceae } \\
\qquad \text { Anthurium minarum Sakur. \& Mayo }\end{array}$ & 1287 & $\mathrm{He}$ & 0.06 & 0.03 & 0.01 & 0 & 0 & 0 \\
\hline Philodendron rhizomatosum Sakur. \& Maio & 1604 & $\mathrm{Cr}$ & 0 & 0.01 & 0 & 0 & 0 & 0 \\
\hline $\begin{array}{l}\text { Araliaceae } \\
\quad \text { Schefflera morototoni (Aubl.) Maguire et al. }\end{array}$ & 1548 & $\mathrm{Ph}$ & 0.05 & 0.15 & 0.35 & 0 & 0 & 0.08 \\
\hline $\begin{array}{l}\text { Aristolochiaceae } \\
\text { Aristolochia fimbriata Cham. }\end{array}$ & 1673 & $\mathrm{He}$ & 0 & 0.01 & 0.01 & 0 & 0 & 0 \\
\hline Aristolochia smilacina (Klotzsch) Duch. & 1667 & $\mathrm{He}$ & 0 & 0.01 & 0.02 & 0.02 & 0 & 0.04 \\
\hline Aristolochia $\mathrm{sp} .1$ & 1664 & $\mathrm{He}$ & 0 & 0 & 0.02 & 0 & 0 & 0 \\
\hline Aristolochia sp.2 & 1859 & $\mathrm{He}$ & 0.06 & 0.01 & 0 & 0.11 & 0.01 & 0 \\
\hline $\begin{array}{l}\text { Asteraceae } \\
\quad \text { Achyrocline satureioides (Lam.) DC. }\end{array}$ & 2132 & $\mathrm{Ph}$ & 0 & 0.01 & 0 & 0 & 0 & 0 \\
\hline Baccharis platypoda $\mathrm{DC}$. & 1974 & $\mathrm{Ph}$ & 0 & 0.03 & 0.02 & 0.13 & 1.79 & 0.01 \\
\hline Baccharis punctulata DC. & 1792 & $\mathrm{Ph}$ & 0 & 0.05 & 0.02 & 0 & 0 & 0 \\
\hline Baccharis reticularia DC. & 1485 & $\mathrm{Ph}$ & 2.12 & 0.34 & 0.79 & 0.47 & 0.11 & 0.79 \\
\hline Baccharis serrulata (Lam.) Pers. & 1851 & $\mathrm{Ph}$ & 0 & 0 & 0 & 0.23 & 0.04 & 0.02 \\
\hline Calea clematidea Baker & 1833 & $\mathrm{Ph}$ & 0 & 0 & 0 & 0.03 & 0.51 & 1.27 \\
\hline Chromolaena squalida (DC.) R.M.King \& H.Rob. & 1487 & $\mathrm{Ph}$ & 0.06 & 0.02 & 0.02 & 0 & 0 & 0 \\
\hline Cyrtocymura scorpioides (Lam.) H.Rob. & 1894 & $\mathrm{Ph}$ & 0 & 0 & 0.02 & 0.05 & 0.24 & 0.11 \\
\hline Dasyphyllum sprengelianum (Gardner) Cabrera & 1740 & $\mathrm{Ph}$ & 0.23 & 0 & 0 & 0 & 0 & 0 \\
\hline Echinocoryne holosericea (Mart. ex DC.) H.Rob. & 1726 & $\mathrm{Ph}$ & 0 & 0.05 & 0.15 & 0 & 0 & 0 \\
\hline Eremanthus crotonoides (DC.) Sch.Bip. & 2117 & $\mathrm{Ph}$ & 0.4 & 1.65 & 2.49 & 5.7 & 5.6 & 5.7 \\
\hline Eremanthus erythropapus (DC.) MacLeish & 1475 & $\mathrm{Ph}$ & 0.6 & 0.8 & 11.8 & 2.8 & 1.16 & 8.25 \\
\hline Eremanthus incanus (Less.) Less. & 1394 & $\mathrm{Ph}$ & 2.5 & 0 & 2.5 & 5.6 & 0.15 & 0 \\
\hline Hololepis penduculata (DC. ex Pers.) DC. & 1383 & $\mathrm{Ph}$ & 0.66 & 0.08 & 0.09 & 0 & 0 & 0 \\
\hline Koanophyllon adamantium (Gardner) R.M.King \& H.Rob & 1653 & $\mathrm{Ph}$ & 0 & 0.02 & 0.11 & 0 & 0 & 0 \\
\hline Lychnophora pinaster Mart. & 1360 & $\mathrm{Ph}$ & 2.23 & 12.3 & 0.5 & 0 & 0 & 0 \\
\hline Lychnophora syncephala (Sch.Bip.) Sch.Bip. & 1496 & $\mathrm{Ph}$ & 0.03 & 0.01 & 0 & 0 & 0 & 0 \\
\hline
\end{tabular}

Voucher: Collect number of MCTB Messias; LF = Life-form; Ph = phanerophyte; He = hemicryptophyte; Cr = cryptophyte; Ch = chamaephyte; $\mathrm{Th}=$ therophyte; ISG = Grasslands in sloped areas, with bare itabirite rocks, near the mountain summit; IPG = Grasslands on the itabirite plateaus, in the middle of the slope, with no more than a $10 \%$ inclination; IWS = Woody savannas in the lower and/or concave parts of the slopes, in valleys or depressions of the itabirite areas; QSG = Grasslands in sloped areas, with bare quartzite rocks, near the mountain summit; QPG = Grasslands on the quartzite plateaus, in the middle of the slope, with no more than a $10 \%$ inclination; QWS = Woody savannas in the lower and concave parts of the slopes, in valleys or depressions of the quartzite areas. 
Table 1. Continued..

\begin{tabular}{|c|c|c|c|c|c|c|c|c|}
\hline \multirow[t]{2}{*}{ Family/Species } & \multirow[t]{2}{*}{ Voucher } & \multirow[t]{2}{*}{$\mathbf{L F}$} & \multicolumn{3}{|c|}{ Itabirite } & \multicolumn{3}{|c|}{ Quartzite } \\
\hline & & & ISG & IPG & IWS & QSG & QPG & QWS \\
\hline Mikania sp. & 1982 & $\mathrm{Li}$ & 0 & 0 & 0 & 0 & 0.06 & 0.05 \\
\hline Moquinia racemosa (Spreng.) DC. & 1651 & $\mathrm{Ph}$ & 0 & 0 & 0.3 & 0 & 0 & 0 \\
\hline $\begin{array}{l}\text { Pseudobrickellia angustissima (Spreng. ex Baker) R.M. } \\
\text { King \& H.Rob. }\end{array}$ & 1454 & $\mathrm{Ph}$ & 0.06 & 0 & 0 & 0 & 0 & 0 \\
\hline Richterago amplexifolia (Gardner) Kuntze & 1714 & $\mathrm{He}$ & 0 & 0 & 0.04 & 0 & 0 & 0 \\
\hline Richterago radiata (Vell.) Roque & 1779 & $\mathrm{He}$ & 0 & 0 & 0 & 0.12 & 0.25 & 0.16 \\
\hline Senecio pohlii Sch.Bip. ex Baker & 1666 & $\mathrm{Ph}$ & 0.01 & 0 & 0.02 & 0 & 0 & 0 \\
\hline Trichogonia hirtiflora (DC.) Sch.Bip. ex Baker & 1720 & $\mathrm{Ch}$ & 0.01 & 0 & 0.02 & 0 & 0 & 0 \\
\hline Trichogonia villosa (Spreng.) Sch.Bip. ex Baker & 1526 & $\mathrm{Ph}$ & 0 & 0 & 0 & 0.02 & 0.04 & 0.02 \\
\hline Vernonia sp. & 1988 & $\mathrm{Ph}$ & 0 & 0 & 0 & 0 & 0.07 & 0.2 \\
\hline $\begin{array}{l}\text { Bignoniaceae } \\
\qquad \text { Handroanthus albus (Cham.) Mattos }\end{array}$ & 1981 & $\mathrm{Ph}$ & 0 & 0 & 0 & 0 & 0.05 & 0.05 \\
\hline $\begin{array}{l}\text { Bromeliaceae } \\
\quad \text { Cryptanthus schwackeanus Mez. }\end{array}$ & 1705 & $\mathrm{He}$ & 0.02 & 0.03 & 0.03 & 0 & 0 & 0 \\
\hline Dyckia cf. cinerea $\mathrm{Mez}$ & 1879 & $\mathrm{He}$ & 0.04 & 0 & 0 & 0.31 & 0 & 0.06 \\
\hline Dyckia rariflora Schult. \& Schult.f. & 1689 & $\mathrm{He}$ & 0.32 & 0.16 & 0.08 & 0.83 & 0 & 0 \\
\hline Tilandsia stricta Sol. & 2168 & Ep & 0 & 0 & 0.01 & 0 & 0 & 0 \\
\hline Vriesea minarum L.B.Sm. & 1671 & $\mathrm{He}$ & 0 & 0.1 & 0.01 & 0 & 0 & 0 \\
\hline $\begin{array}{l}\text { Chrysobalanaceae } \\
\text { Hirtella floribunda Cham. \& Schltdl. }\end{array}$ & 2032 & $\mathrm{Li}$ & 0 & 0 & 0 & 0 & 0.02 & 0 \\
\hline $\begin{array}{l}\text { Clusiaceae } \\
\quad \text { Kielmeyera coriacea Mart. \& Zucc. }\end{array}$ & 1783 & $\mathrm{Ph}$ & 0 & 0 & 0 & 0 & 0.02 & 0 \\
\hline $\begin{array}{l}\text { Convolvulaceae } \\
\quad \text { Jacquemontia prostrata } \text { Choisy }\end{array}$ & 1868 & $\mathrm{He}$ & 0 & 0 & 0.02 & 0 & 0 & 0 \\
\hline $\begin{array}{l}\text { Cyperaceae } \\
\quad \text { Bulbostylis capillaris (L.) C.B.Clarke }\end{array}$ & 1928 & $\mathrm{He}$ & 0 & 0 & 0 & 0.03 & 0.02 & 0.02 \\
\hline Bulbostylis juncoides (Vahl) Kük. & 1924 & $\mathrm{He}$ & 0.03 & 0 & 0 & 0.09 & 0.02 & 0 \\
\hline Bulbostylis sp. & 2016 & $\mathrm{He}$ & 0 & 0 & 0 & 0 & 0.01 & 0 \\
\hline Dichromena sp. & 1598 & $\mathrm{He}$ & 0 & 0.01 & 0 & 0 & 0 & 0 \\
\hline Lagenocarpus rigidus Ness & 1292 & $\mathrm{He}$ & 22 & 4.45 & 3.15 & 7.76 & 1.07 & 0.03 \\
\hline Rhynchospora corymbosa (L.) Britton & 2031 & $\mathrm{He}$ & 0 & 0 & 0 & 0 & 0 & 0.4 \\
\hline Rhynchospora tenuis Link & 1940 & $\mathrm{He}$ & 0 & 0.12 & 0 & 0.02 & 0.13 & 0.14 \\
\hline Rhynchospora sp.1 & 1930 & $\mathrm{He}$ & 0.5 & 0.51 & 0 & 0 & 0 & 0.02 \\
\hline Rhynchospora sp.2 & 2093 & $\mathrm{He}$ & 0 & 0 & 0 & 0 & 0.06 & 0 \\
\hline Scleria hirtella Sw. & 1886 & $\mathrm{He}$ & 0 & 0 & 0 & 0.01 & 0.11 & 0.05 \\
\hline Scleria sp. & 1644 & $\mathrm{He}$ & 0 & 0.02 & 0.01 & 0 & 0 & 0 \\
\hline Trilepis microstachya (C.B.Clarke) H.Pfeiff. & 1594 & $\mathrm{He}$ & 0.01 & 0.21 & 0.03 & 0 & 0 & 0 \\
\hline $\begin{array}{l}\text { Dilleniaceae } \\
\qquad \text { Doliocarpus elegans Eichler }\end{array}$ & 2274 & $\mathrm{Li}$ & 0 & 0 & 0 & 0 & 0.1 & 0 \\
\hline $\begin{array}{l}\text { Dioscoreaceae } \\
\quad \text { Dioscorea campestris Griseb. }\end{array}$ & 1875 & $\mathrm{Cr}$ & 0.02 & 0 & 0 & 0.02 & 0 & 0.01 \\
\hline Dioscorea debilis Uline ex R.Knuth & 1675 & $\mathrm{Cr}$ & 0 & 0.03 & 0.05 & 0 & 0 & 0 \\
\hline $\begin{array}{l}\text { Ericaceae } \\
\quad \text { Agarista pulchella var. cordifolia (Meisn.) Judd }\end{array}$ & 1421 & $\mathrm{Ph}$ & 0.18 & 0.15 & 0 & 0 & 0 & 0 \\
\hline Agarista pulchra (Cham. \& Schltdl.) G.Don & 1716 & $\mathrm{Ph}$ & 0 & 0 & 0.02 & 0 & 0 & 0 \\
\hline Gaylussacia amoena Cham. & 1650 & $\mathrm{Ph}$ & 0 & 0 & 0.03 & 0 & 0 & 0 \\
\hline Gaylussacia reticulata Mart. ex Meisn. var. reticulata & 1670 & $\mathrm{Ph}$ & 0 & 0.1 & 0 & 0 & 0 & 0 \\
\hline
\end{tabular}

Voucher: Collect number of MCTB Messias; LF = Life-form; $\mathrm{Ph}=$ phanerophyte; $\mathrm{He}=$ hemicryptophyte; $\mathrm{Cr}=$ cryptophyte; Ch = chamaephyte; $\mathrm{Th}=$ therophyte; ISG = Grasslands in sloped areas, with bare itabirite rocks, near the mountain summit; IPG = Grasslands on the itabirite plateaus, in the middle of the slope, with no more than a $10 \%$ inclination; IWS = Woody savannas in the lower and/or concave parts of the slopes, in valleys or depressions of the itabirite areas; QSG = Grasslands in sloped areas, with bare quartzite rocks, near the mountain summit; QPG = Grasslands on the quartzite plateaus, in the middle of the slope, with no more than a $10 \%$ inclination; QWS = Woody savannas in the lower and concave parts of the slopes, in valleys or depressions of the quartzite areas. 
Messias, M.C.T.B.

Table 1. Continued...

\begin{tabular}{|c|c|c|c|c|c|c|c|c|}
\hline \multirow[t]{2}{*}{ Family/Species } & \multirow[t]{2}{*}{ Voucher } & \multirow[t]{2}{*}{$\mathbf{L F}$} & \multicolumn{3}{|c|}{ Itabirite } & \multicolumn{3}{|c|}{ Quartzite } \\
\hline & & & ISG & IPG & IWS & QSG & QPG & QWS \\
\hline Eriocaulaceae & 1796 & $\mathrm{He}$ & 0 & 0 & 0.02 & 0 & 0 & 0 \\
\hline Actinocephalus bongardii (A.St.-Hil.) Sano & & & & & & & & \\
\hline Paepalanthus decussus Körn. & 1963 & $\mathrm{He}$ & 0 & 0 & 0 & 0 & 0.01 & 0 \\
\hline Paepalanthus dianthoides Mart. ex Körn. & 1733 & $\mathrm{He}$ & 0 & 0 & 0.03 & 0 & 0 & 0 \\
\hline Paepalanthus cacuminis Ruhland & 1719 & $\mathrm{He}$ & 0 & 0 & 0.04 & 0 & 0 & 0 \\
\hline Paepalanthus planifolius (Bong.) Körn. & 1524 & $\mathrm{He}$ & 0.01 & 0 & 0.01 & 0.02 & 0.89 & 0.15 \\
\hline Paepalanthus vaginatus Körn. & 1861 & $\mathrm{He}$ & 0 & 0 & 0 & 0.08 & 0.42 & 0.2 \\
\hline $\begin{array}{l}\text { Erythroxylaceae } \\
\text { Erythroxylum gonocladum (Mart.) O.E.Schulz }\end{array}$ & 1652 & $\mathrm{Ph}$ & 0 & 0 & 0.5 & 0 & 0 & 0 \\
\hline $\begin{array}{l}\text { Euphorbiaceae } \\
\quad \text { Croton comosus Müll.Arg. }\end{array}$ & 1478 & $\mathrm{Ph}$ & 0.08 & 0.02 & 0 & 0 & 0 & 0 \\
\hline Croton erythroxyloides Baill. & 1397 & $\mathrm{Ph}$ & 0.90 & 0.99 & 0.1 & 0 & 0 & 0 \\
\hline Maprounea brasiliensis A.St.-Hil. & 1513 & $\mathrm{Ph}$ & 0 & 0 & 0 & 0 & 0 & 0.2 \\
\hline Microstachys daphnoides (Mart.) Müll. Arg. & 1596 & $\mathrm{Ph}$ & 0 & 0.02 & 0.08 & 0 & 0 & 0 \\
\hline $\begin{array}{l}\text { Fabaceae (Leguminosae - Caesalpinoideae) } \\
\text { Bauhinia rufa (Bong.) Steud. }\end{array}$ & 2013 & $\mathrm{Ph}$ & 0.9 & 0.12 & 0.05 & 0.02 & 0 & 0 \\
\hline Chamaecrista desvauxii (Collad.) Killip & 1993 & $\mathrm{Ch}$ & 0 & 0 & 0 & 0 & 0.02 & 0 \\
\hline Chamaecrista flexuosa (L.) Greene & 1836 & $\mathrm{Ch}$ & 0 & 0 & 0 & 0.05 & 0.05 & 0.01 \\
\hline Chamaecrista mucronata (Spreng.) H.S.Irwin \& Barneby & 1632 & $\mathrm{Ph}$ & 0 & 0 & 0.04 & 0.01 & 0.1 & 0.06 \\
\hline Chamaecrista ochnacea (Vogel) H.S.Irwin \& Barneby & 1828 & $\mathrm{Ph}$ & 0 & 0 & 0 & 0 & 0 & 0.02 \\
\hline Chamaecrista rotundifolia (Pers.) Greene & 1891 & $\mathrm{He}$ & 0 & 0 & 0 & 0.01 & 0 & 0.01 \\
\hline Senna reniformis (G. Don) H.S.Irwin \& Barneby & 1473 & $\mathrm{Ph}$ & 0.1 & 0 & 0 & 0 & 0 & 0.06 \\
\hline $\begin{array}{l}\text { Fabaceae (Leguminosae - Papilionoideae) } \\
\text { Aeschynomene elegans Schltdl. \& Cham. }\end{array}$ & 1558 & $\mathrm{Ch}$ & 0.01 & 0.02 & 0.03 & 0 & 0 & 0.01 \\
\hline Centrosema coriaceum Benth. & 1818 & $\mathrm{He}$ & 0.06 & 0.02 & 0.05 & 0 & 0 & 0 \\
\hline Clitoria densiflora (Benth.) Benth. & 1702 & $\mathrm{Ph}$ & 0 & 0 & 0.01 & 0 & 0 & 0 \\
\hline Desmodium barbatum (L.) Benth. & 1913 & $\mathrm{He}$ & 0 & 0 & 0 & 0.01 & 0 & 0.01 \\
\hline Galactia martii DC. & 1384 & $\mathrm{He}$ & 0.12 & 0.05 & 0 & 0 & 0 & 0 \\
\hline Periandra mediterranea (Vell.) Taub. & 1522 & $\mathrm{Ph}$ & 1.48 & 0.37 & 0.59 & 0.77 & 0.18 & 0.43 \\
\hline Stylosanthes gracilis Kunth & 1965 & $\mathrm{Ch}$ & 0 & 0 & 0 & 0.01 & 0.01 & 0.01 \\
\hline Stylosanthes ruellioides Mart. & 1694 & $\mathrm{Ch}$ & 0 & 0 & 0.05 & 0 & 0 & 0 \\
\hline Stylosanthes viscosa (L.) Sw. & 1911 & $\mathrm{Ch}$ & 0 & 0 & 0 & 0.01 & 0.01 & 0.03 \\
\hline Zornia reticulata $\mathrm{Sm}$. & 1889 & $\mathrm{Ch}$ & 0 & 0 & 0 & 0.03 & 0.01 & 0.02 \\
\hline $\begin{array}{l}\text { Gentianaceae } \\
\quad \text { Calolisianthus pedunculatus (Cham. \& Schltdl.) Gilg }\end{array}$ & 2162 & $\mathrm{Ch}$ & 0 & 0 & 0.01 & 0 & 0 & 0 \\
\hline $\begin{array}{l}\text { Gesneriaceae } \\
\quad \text { Nematanthus strigillosus (Mart.) H.E. Moore }\end{array}$ & 1621 & $\mathrm{Ch}$ & 0.01 & 0.01 & 0.06 & 0 & 0 & 0 \\
\hline Paliavana sericiflora Benth. & 1464 & $\mathrm{Ph}$ & 0.38 & 0.15 & 0.22 & 0 & 0 & 0 \\
\hline $\begin{array}{l}\text { Gleicheniaceae } \\
\quad \text { Dicranopteris flexuosa (Schrad.) Underw. }\end{array}$ & 1915 & $\mathrm{He}$ & 0 & 0 & 0 & 0.01 & 0.53 & 0 \\
\hline $\begin{array}{l}\text { Hypericaceae } \\
\quad \text { Vismia brasiliensis Choisy }\end{array}$ & 2247 & $\mathrm{Ph}$ & 0 & 0 & 0 & 0 & 0.1 & 0 \\
\hline $\begin{array}{l}\text { Iridaceae } \\
\qquad \text { Cipura paludosa Aubl. }\end{array}$ & 1914 & $\mathrm{He}$ & 0 & 0 & 0 & 0.01 & 0 & 0 \\
\hline Neomarica sp. & 1611 & $\mathrm{Cr}$ & 0.01 & 0.02 & 0 & 0 & 0 & 0.01 \\
\hline Sisyrinchium sp. & 1625 & $\mathrm{Cr}$ & 0.01 & 0.01 & 0.04 & 0 & 0 & 0 \\
\hline Trimezia sp. & 1674 & $\mathrm{Cr}$ & 0 & 0.01 & 0.03 & 0 & 0 & 0 \\
\hline
\end{tabular}

Voucher: Collect number of MCTB Messias; LF = Life-form; Ph = phanerophyte; He = hemicryptophyte; $\mathrm{Cr}=$ cryptophyte; Ch = chamaephyte; $\mathrm{Th}=$ therophyte; $\mathrm{ISG}=$ Grasslands in sloped areas, with bare itabirite rocks, near the mountain summit; IPG = Grasslands on the itabirite plateaus, in the middle of the slope, with no more than a $10 \%$ inclination; IWS = Woody savannas in the lower and/or concave parts of the slopes, in valleys or depressions of the itabirite areas; QSG = Grasslands in sloped areas, with bare quartzite rocks, near the mountain summit; QPG = Grasslands on the quartzite plateaus, in the middle of the slope, with no more than a $10 \%$ inclination; QWS = Woody savannas in the lower and concave parts of the slopes, in valleys or depressions of the quartzite areas. 
Table 1. Continued...

\begin{tabular}{|c|c|c|c|c|c|c|c|c|}
\hline \multirow[t]{2}{*}{ Family/Species } & \multirow[t]{2}{*}{ Voucher } & \multirow[t]{2}{*}{$\mathbf{L F}$} & \multicolumn{3}{|c|}{ Itabirite } & \multicolumn{3}{|c|}{ Quartzite } \\
\hline & & & ISG & IPG & IWS & QSG & QPG & QWS \\
\hline $\begin{array}{l}\text { Lamiaceae } \\
\quad \text { Aegiphila verticillata Vell. }\end{array}$ & 1580 & $\mathrm{Ph}$ & 0 & 0 & 0.15 & 0 & 0 & 0 \\
\hline Hyptis homalophylla Pohl ex Benth. & 1895 & $\mathrm{Ch}$ & 0 & 0 & 0.03 & 0.03 & 0.14 & 0.16 \\
\hline Hyptis monticola Mart.ex Benth. & 1685 & $\mathrm{Ph}$ & 0.7 & 0.27 & 0.07 & 0 & 0 & 0 \\
\hline Hyptis passerina Mart.ex Benth. & 1451 & $\mathrm{Ph}$ & 0.17 & 0 & 0 & 0 & 0 & 0 \\
\hline $\begin{array}{l}\text { Lauraceae } \\
\quad \text { Cinnamomum erythropus (Nees \& Mart.) Kosterm. }\end{array}$ & 1790 & $\mathrm{Ph}$ & 0 & 0.1 & 0.04 & 0 & 0 & 0 \\
\hline Ocotea caesia $\mathrm{Mez}$ & 1636 & $\mathrm{Ph}$ & 0.15 & 0 & 0.93 & 0 & 0 & 0 \\
\hline Ocotea nutans (Nees) Mez & 1729 & $\mathrm{Ph}$ & 0 & 0.3 & 0.18 & 0 & 0 & 0.08 \\
\hline Ocotea percoriacea Kosterm. & 1711 & $\mathrm{Ph}$ & 0 & 0 & 0.54 & 0 & 0 & 0 \\
\hline Persea sp. & 1718 & $\mathrm{Ph}$ & 0 & 0 & 0.02 & 0 & 0 & 0 \\
\hline $\begin{array}{l}\text { Lentibulariaceae } \\
\quad \text { Utricularia amethystina Salzm. ex A.St.-Hil. \& Girard }\end{array}$ & 1983 & Th & 0 & 0 & 0 & 0 & 0.01 & 0 \\
\hline $\begin{array}{l}\text { Lindsaeaceae } \\
\quad \text { Lindsaea guianensis (Aubl.) Dryand. }\end{array}$ & 2063 & $\mathrm{He}$ & 0 & 0 & 0 & 0 & 0 & 0.01 \\
\hline $\begin{array}{l}\text { Loganiaceae } \\
\quad \text { Spigelia spartioides Cham. }\end{array}$ & 1631 & Th & 0 & 0.01 & 0.03 & 0 & 0 & 0 \\
\hline $\begin{array}{l}\text { Loranthaceae } \\
\quad \text { Phoradendron falcifrons (Hook. \& Arn.) Eichler }\end{array}$ & 1978 & $\mathrm{Li}$ & 0 & 0 & 0 & 0 & 0.08 & 0.04 \\
\hline $\begin{array}{l}\text { Lycopodiaceae } \\
\quad \text { Lycopodiella cernua (L.) Pic.Serm. }\end{array}$ & 2025 & $\mathrm{He}$ & 0 & 0 & 0 & 0 & 0.01 & 0 \\
\hline $\begin{array}{l}\text { Lythraceae } \\
\quad \text { Diplusodon microphyllus Pohl }\end{array}$ & 1704 & $\mathrm{Ph}$ & 0.44 & 0.21 & 0.2 & 0 & 0 & 0 \\
\hline $\begin{array}{l}\text { Malpighiaceae } \\
\quad \text { Byrsonima variabilis A.Juss. }\end{array}$ & 1633 & $\mathrm{Ph}$ & 1.28 & 0.08 & 1.69 & 0.3 & 0 & 0.33 \\
\hline Heteropterys campestris A.Juss. & 1787 & $\mathrm{Ph}$ & 0.28 & 0.17 & 0.2 & 0 & 0 & 0 \\
\hline Heteropterys escalloniifolia A.Juss. & 1671 & $\mathrm{Ph}$ & 0.07 & 0.01 & 0.06 & 0 & 0 & 0 \\
\hline Heteropterys sp. & 2062 & $\mathrm{Ph}$ & 0 & 0 & 0 & 0.2 & 0.15 & 0.56 \\
\hline $\begin{array}{l}\text { Malvaceae } \\
\quad \text { Sida linifolia } \text { Cav. }\end{array}$ & 1896 & $\mathrm{Ch}$ & 0 & 0 & 0 & 0.03 & 0.01 & 0.03 \\
\hline $\begin{array}{l}\text { Melastomataceae } \\
\qquad \text { Acisanthera variabilis (Mart. \& Schrank) Triana }\end{array}$ & 1684 & $\mathrm{Ph}$ & 0 & 0 & 0.02 & 0 & 0 & 0 \\
\hline Cambessedesia hilariana (Kunth) DC. & 1831 & $\mathrm{Ch}$ & 0 & 0 & 0 & 0.06 & 0.09 & 0.05 \\
\hline Clidemia urceolata DC. & 2206 & $\mathrm{Ph}$ & 0 & 0 & 0 & 3.85 & 6.05 & 4.35 \\
\hline Leandra aurea (Cham.) Cogn. & 1583 & $\mathrm{Ph}$ & 0 & 0 & 0.02 & 0 & 0 & 0 \\
\hline Leandra australis (Cham.) Cogn. & 1709 & $\mathrm{Ph}$ & 0.84 & 0.42 & 1.48 & 1.63 & 0 & 0 \\
\hline Leandra dendroides (Naudin) Cogn. & 2021 & $\mathrm{Ph}$ & 0 & 0.05 & 0.02 & 0 & 0 & 0 \\
\hline Leandra foveolata (DC.) Cogn. & 1977 & $\mathrm{Ph}$ & 0 & 0 & 0 & 0 & 0.06 & 0 \\
\hline Miconia albicans (Sw.) Triana & 1530 & $\mathrm{Ph}$ & 0 & 0 & 0 & 0.21 & 0.37 & 0.63 \\
\hline Miconia corallina Spring & 1794 & $\mathrm{Ph}$ & 0 & 0 & 0.08 & 0 & 0 & 0.03 \\
\hline Miconia ligustroides (DC.) Naudin & 1703 & $\mathrm{Ph}$ & 0 & 0 & 0.4 & 0 & 0 & 0.1 \\
\hline Miconia stenostachya DC. & 1873 & $\mathrm{Ph}$ & 0 & 0 & 0 & 0.02 & 0.03 & 0.05 \\
\hline Microlicia crenulata (DC.) Mart. & 1551 & $\mathrm{Ph}$ & 0 & 0.32 & 0 & 0 & 0 & 0 \\
\hline Microlicia fulva (Spreng.) Cham. & 1964 & $\mathrm{Ph}$ & 0 & 0.02 & 0 & 0 & 0.11 & 0 \\
\hline Microlicia graveolens DC. & 1883 & $\mathrm{Ph}$ & 0 & 0 & 0 & 0.2 & 0.29 & 0.04 \\
\hline Tibouchina gardneriana (Triana) Cogn. & 1550 & $\mathrm{Ph}$ & 0.02 & 0.03 & 0.05 & 0 & 0 & 0 \\
\hline Tibouchina heteromalla (D.Don) Cogn. & 1367 & $\mathrm{Ph}$ & 4.25 & 2.16 & 1.61 & 0.31 & 0.37 & 1.64 \\
\hline Trembleya laniflora (D.Don) Cogn. & 1922 & $\mathrm{Ph}$ & 0 & 0 & 2.7 & 0.31 & 0.06 & 0.05 \\
\hline
\end{tabular}

Voucher: Collect number of MCTB Messias; LF = Life-form; $\mathrm{Ph}=$ phanerophyte; $\mathrm{He}=$ hemicryptophyte; $\mathrm{Cr}=$ cryptophyte; Ch = chamaephyte; $\mathrm{Th}=$ therophyte; ISG = Grasslands in sloped areas, with bare itabirite rocks, near the mountain summit; IPG = Grasslands on the itabirite plateaus, in the middle of the slope, with no more than a $10 \%$ inclination; IWS = Woody savannas in the lower and/or concave parts of the slopes, in valleys or depressions of the itabirite areas; QSG = Grasslands in sloped areas, with bare quartzite rocks, near the mountain summit; $\mathrm{QPG}=$ Grasslands on the quartzite plateaus, in the middle of the slope, with no more than a $10 \%$ inclination; QWS = Woody savannas in the lower and concave parts of the slopes, in valleys or depressions of the quartzite areas. 
Table 1. Continued...

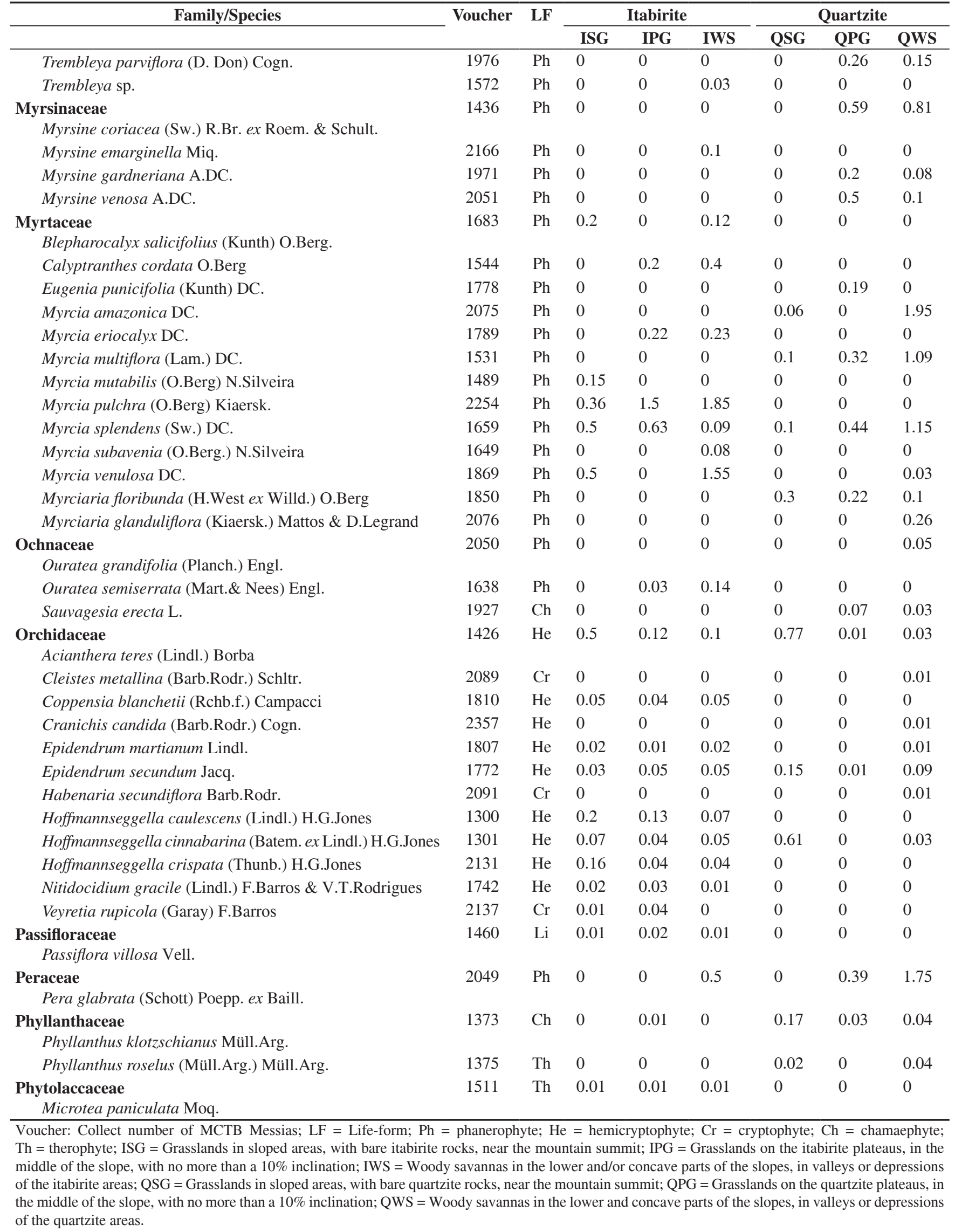


Table 1. Continued...

\begin{tabular}{|c|c|c|c|c|c|c|c|c|}
\hline \multirow[t]{2}{*}{ Family/Species } & \multirow[t]{2}{*}{ Voucher } & \multirow[t]{2}{*}{$\mathbf{L F}$} & \multicolumn{3}{|c|}{ Itabirite } & \multicolumn{3}{|c|}{ Quartzite } \\
\hline & & & ISG & IPG & IWS & QSG & QPG & QWS \\
\hline $\begin{array}{l}\text { Poaceae } \\
\quad \text { Andropogon bicornis } \mathrm{L} \text {. }\end{array}$ & 2042 & $\mathrm{He}$ & 0 & 0 & 0 & 0 & 0.07 & 0.07 \\
\hline Andropogon leucostachyus Kunth & 1588 & $\mathrm{He}$ & 0 & 0.02 & 0 & 0 & 0 & 0 \\
\hline Andropogon selloanus (Hack.) Hack. & 1935 & $\mathrm{He}$ & 0 & 0 & 0 & 0.04 & 0.06 & 0.04 \\
\hline Andropogon virgatus Desv. & 1947 & $\mathrm{He}$ & 0 & 0 & 0 & 0 & 0.05 & 0.03 \\
\hline $\begin{array}{l}\text { Apochloa poliophylla (Renvoize \& Zuloaga) Zuloaga \& } \\
\text { Morrone }\end{array}$ & 1566 & $\mathrm{He}$ & 5.67 & 5.66 & 3.02 & 1.9 & 0.08 & 0.94 \\
\hline Aristida torta (Nees) Kunth & 1884 & $\mathrm{He}$ & 0 & 0 & 0 & 0.09 & 0.1 & 0.08 \\
\hline Axonopus capillaris (Lam.) Chase & 1907 & $\mathrm{He}$ & 0 & 0 & 0 & 0.01 & 0.02 & 0 \\
\hline Axonopus laxiflorus (Trin.) Chase & 1623 & $\mathrm{He}$ & 1.13 & 0.52 & 0.59 & 0.76 & 0.03 & 6.54 \\
\hline Axonopus scoparius (Flüggé) Kuhlm. & 1445 & $\mathrm{He}$ & 0 & 0 & 0 & 0 & 0.01 & 0 \\
\hline Axonopus siccus (Nees) Kuhlm. & 1852 & $\mathrm{He}$ & 0.5 & 1.54 & 0.21 & 0.26 & 0.14 & 0.08 \\
\hline Dichantelium sciurotoides (Zuloaga \& Morrone) Davidse & 2037 & $\mathrm{He}$ & 0 & 0 & 0 & 0 & 0 & 0.06 \\
\hline Echinolaena inflexa (Poir.) Chase & 1863 & $\mathrm{He}$ & 0 & 0 & 0 & 2.41 & 11.9 & 9.1 \\
\hline Eragrostis rufescens Schrad. ex Schult. & 1929 & $\mathrm{He}$ & 0 & 0 & 0 & 0 & 0.01 & 0 \\
\hline Eragrostis solida Nees & 1892 & $\mathrm{He}$ & 0 & 0 & 0 & 0.01 & 0.02 & 0.03 \\
\hline Ichnanthus bambusiflorus (Trin.) Döll & 1467 & $\mathrm{He}$ & 0.02 & 0.54 & 0.26 & 0 & 0 & 0.04 \\
\hline Otachyrium versicolor (Döll) Henrard & 1949 & $\mathrm{He}$ & 0 & 0 & 0 & 0 & 0.07 & 0.12 \\
\hline Panicum pseudisachne $\mathrm{Mez}$ & 1672 & $\mathrm{He}$ & 0.04 & 0.04 & 0.64 & 0.03 & 0.03 & 0.08 \\
\hline Panicum wettsteinii Hack. & 1549 & $\mathrm{He}$ & 0 & 0.65 & 0.62 & 0.02 & 0 & 0 \\
\hline $\begin{array}{l}\text { Parodiophyllochloa penicillata (Nees ex Trin.) } \\
\text { Zuloaga \& Morrone }\end{array}$ & 2060 & $\mathrm{He}$ & 0 & 0 & 0 & 0 & 0 & 0.02 \\
\hline Paspalum coryphaeum Trin. & 2084 & $\mathrm{He}$ & 0 & 0 & 0 & 0 & 0 & 0.01 \\
\hline Paspalum hyalinum Nees ex Trin. & 1909 & $\mathrm{He}$ & 0 & 0 & 0 & 0 & 0.09 & 0 \\
\hline Paspalum lineare Trin. & 1878 & $\mathrm{He}$ & 0 & 0 & 0 & 0.04 & 0.02 & 0.01 \\
\hline Paspalum multicaule Poir. & 1897 & $\mathrm{He}$ & 0 & 0 & 0 & 0.01 & 0 & 0 \\
\hline Paspalum pilosum Lam. & 1921 & $\mathrm{He}$ & 0 & 0 & 0 & 0.03 & 0.12 & 0.08 \\
\hline Paspalum polyphyllum Nees & 1999 & $\mathrm{He}$ & 0 & 0 & 0 & 0.09 & 0.02 & 0.04 \\
\hline Schizachyrium sanguineum (Retz.) Alston & 1961 & $\mathrm{He}$ & 0.25 & 0.35 & 0.02 & 0.02 & 0.07 & 0.02 \\
\hline Schizachyrium tenerum Nees & 1916 & $\mathrm{He}$ & 1.29 & 0.61 & 0.23 & 0.05 & 0.01 & 0.16 \\
\hline Sporobolus metallicolus Longhi-Wagner \& Boechat & 1931 & $\mathrm{He}$ & 0 & 0 & 0 & 0 & 0.03 & 0.09 \\
\hline $\begin{array}{l}\text { Polygalaceae } \\
\quad \text { Polygala cuspidata DC. }\end{array}$ & 2041 & $\mathrm{Ch}$ & 0 & 0 & 0 & 0 & 0 & 0.01 \\
\hline Polygala oleifolia A.St.-Hil. \& Moq. & 1661 & $\mathrm{Ch}$ & 0 & 0 & 0.05 & 0 & 0 & 0 \\
\hline Polygala galioides Poir. & 1887 & $\mathrm{Th}$ & 0 & 0 & 0 & 0.03 & 0.08 & 0.04 \\
\hline Polygala paniculata L. & 1888 & Th & 0 & 0 & 0 & 0.03 & 0.06 & 0.03 \\
\hline $\begin{array}{l}\text { Polygonaceae } \\
\text { Coccoloba acrostichoides Cham. }\end{array}$ & 1438 & $\mathrm{Ph}$ & 1.35 & 4.75 & 3.2 & 0 & 0.1 & 0.03 \\
\hline Coccoloba scandens Casar. & 2011 & $\mathrm{Li}$ & 0 & 0 & 0 & 0 & 0.23 & 0.1 \\
\hline $\begin{array}{l}\text { Polypodiaceae } \\
\quad \text { Pleopeltis hirsutissima (Raddi) de la Sota }\end{array}$ & 1679 & $\mathrm{He}$ & 0 & 0 & 0.01 & 0 & 0 & 0 \\
\hline $\begin{array}{l}\text { Pteridaceae } \\
\quad \text { Doryopteris ornithopus (Hook. \& Baker) J.Sm. }\end{array}$ & 1798 & $\mathrm{He}$ & 0.01 & 0.03 & 0.04 & 0.01 & 0 & 0 \\
\hline Pellaea crenata R.M.Tryon & 1717 & $\mathrm{He}$ & 0 & 0 & 0.01 & 0 & 0 & 0 \\
\hline $\begin{array}{l}\text { Rubiaceae } \\
\quad \text { Alibertia rotunda (Cham.) K.Schum. }\end{array}$ & 1866 & $\mathrm{Ph}$ & 0 & 0.07 & 0.08 & 0.02 & 0 & 0.15 \\
\hline Augusta longifolia (Spreng.) Rehder & 1574 & $\mathrm{Ph}$ & 0 & 0 & 0.01 & 0 & 0 & 0 \\
\hline
\end{tabular}


Table 1. Continued...

\begin{tabular}{|c|c|c|c|c|c|c|c|c|}
\hline \multirow[t]{2}{*}{ Family/Species } & \multirow[t]{2}{*}{ Voucher } & \multirow[t]{2}{*}{$\mathbf{L F}$} & \multicolumn{3}{|c|}{ Itabirite } & \multicolumn{3}{|c|}{ Quartzite } \\
\hline & & & ISG & IPG & IWS & QSG & QPG & QWS \\
\hline Borreria poaya (A.St.-Hil.) DC. & 1627 & $\mathrm{Ch}$ & 0 & 0 & 0 & 0 & 0 & 0.02 \\
\hline Borreria sp. & 2086 & $\mathrm{Ch}$ & 0 & 0 & 0 & 0 & 0 & 0.01 \\
\hline Coccocypselum condalia Pers. & 2094 & $\mathrm{Ch}$ & 0 & 0 & 0.05 & 0 & 0.01 & 0.06 \\
\hline Declieuxia fruticosa (Willd. ex Roem. \& Schult.) Kuntze & 1540 & $\mathrm{Ch}$ & 0.01 & 0.03 & 0 & 0 & 0 & 0 \\
\hline Ferdinandusa sp. & 1690 & $\mathrm{Ph}$ & 0 & 0 & 0.01 & 0 & 0 & 0 \\
\hline Mitracarpus hirtus (L.) DC. & 1904 & $\mathrm{Ph}$ & 0 & 0 & 0 & 0.01 & 0 & 0 \\
\hline Palicourea sp. & 2266 & $\mathrm{Ph}$ & 0 & 0 & 0 & 0 & 0 & 0.02 \\
\hline Psychotria hoffmannseggiana (Willd. ex Schult.) Müll.Arg. & 2048 & $\mathrm{Ph}$ & 0 & 0 & 0 & 0 & 0 & 0.08 \\
\hline Psychotria pleiocephala Müll.Arg. & 2068 & $\mathrm{Ph}$ & 0 & 0 & 0 & 0 & 0 & 0.01 \\
\hline Psychotria vellosiana Benth. & 1581 & $\mathrm{Ph}$ & 0 & 0 & 0.02 & 0 & 0 & 0.02 \\
\hline Psyllocarpus laricoides Mart. ex Mart. \& Zucc. & 1402 & $\mathrm{Ch}$ & 0.14 & 0.08 & 0.05 & 0.11 & 0 & 0.08 \\
\hline Remijia ferruginea (A.St.-Hil.) DC. & 1385 & $\mathrm{Ph}$ & 0 & 0 & 0.05 & 0 & 0 & 0 \\
\hline $\begin{array}{l}\text { Rutaceae } \\
\quad \text { Dictyoloma vandellianum A. Juss. }\end{array}$ & 2103 & $\mathrm{Ph}$ & 0 & 0 & 0 & 0.08 & 0 & 0 \\
\hline $\begin{array}{l}\text { Sapindaceae } \\
\qquad \text { Matayba marginata Radlk. }\end{array}$ & 1492 & $\mathrm{Ph}$ & 0.08 & 0 & 0 & 0 & 0 & 0 \\
\hline Serjania gracilis Radlk. & 2036 & $\mathrm{Li}$ & 0 & 0 & 0 & 0 & 0 & 0.11 \\
\hline $\begin{array}{l}\text { Selaginellaceae } \\
\quad \text { Selaginella } \mathrm{sp} .\end{array}$ & 2082 & $\mathrm{He}$ & 0 & 0.01 & 0 & 0 & 0 & 0.01 \\
\hline $\begin{array}{l}\text { Siparunaceae } \\
\quad \text { Siparuna poeppigii (Tul.) A.DC. }\end{array}$ & 2054 & $\mathrm{Ph}$ & 0 & 0 & 0 & 0 & 0 & 0.79 \\
\hline $\begin{array}{l}\text { Smilacaceae } \\
\quad \text { Smilax minarum A.DC. }\end{array}$ & 2020 & $\mathrm{Li}$ & 0 & 0 & 0 & 0.01 & 0 & 0 \\
\hline $\begin{array}{l}\text { Solanaceae } \\
\qquad \text { Schwenckia americana Rooyen ex L. }\end{array}$ & 1776 & Th & 0 & 0 & 0 & 0.05 & 0.01 & 0.02 \\
\hline $\begin{array}{l}\text { Styracaceae } \\
\quad \text { Styrax maninul B.Walln. }\end{array}$ & 1811 & $\mathrm{Ph}$ & 0 & 0.03 & 0.08 & 0 & 0 & 0 \\
\hline $\begin{array}{l}\text { Velloziaceae } \\
\qquad \text { Barbacenia flava Mart. ex Schult. \& Schult.f. }\end{array}$ & 1881 & $\mathrm{He}$ & 0 & 0 & 0 & 0.08 & 0 & 0 \\
\hline Vellozia compacta Mart. ex Schult. \& Schult.f. & 1845 & $\mathrm{Ph}$ & 46 & 19 & 9.3 & 3.65 & 0 & 0.6 \\
\hline Vellozia graminea Pohl & 1736 & $\mathrm{He}$ & 0.01 & 0.14 & 0.09 & 0 & 0 & 0 \\
\hline $\begin{array}{l}\text { Verbenaceae } \\
\quad \text { Lantana fucata Lindl. }\end{array}$ & 1480 & $\mathrm{Ph}$ & 0.02 & 0.04 & 0.04 & 0 & 0 & 0 \\
\hline Lantana trifolia $\mathrm{L}$. & 1557 & $\mathrm{Ph}$ & 0 & 0.02 & 0 & 0 & 0 & 0 \\
\hline Lippia hermannioides Cham. & 1762 & $\mathrm{Ph}$ & 0.05 & 0.08 & 0 & 0 & 0 & 0 \\
\hline Lippia origanoides Kunth & 1450 & $\mathrm{Ph}$ & 0.44 & 0.41 & 0 & 0 & 0 & 0 \\
\hline Lippia rubiginosa Schauer & 1536 & $\mathrm{Ph}$ & 0.1 & 0.5 & 0.22 & 0 & 0 & 0 \\
\hline Stachytarpheta glabra Cham. & 1945 & $\mathrm{Ph}$ & 0.71 & 0.23 & 0.31 & 0.97 & 0.07 & 0.14 \\
\hline $\begin{array}{l}\text { Vochysiaceae } \\
\quad \text { Callisthene major Mart. \& Zucc. }\end{array}$ & 2067 & $\mathrm{Ph}$ & 0 & 0 & 0 & 0 & 0 & 0.08 \\
\hline Vochysia emarginata (Vahl) Poir & 2149 & $\mathrm{Ph}$ & 0 & 0 & 0.28 & 0 & 0 & 0 \\
\hline $\begin{array}{l}\text { Xyridaceae } \\
\quad \text { Xyris sp.1 }\end{array}$ & 1966 & $\mathrm{He}$ & 0 & 0 & 0 & 0 & 0.02 & 0 \\
\hline Xyris sp. 2 & 1942 & $\mathrm{He}$ & 0 & 0 & 0 & 0 & 0.04 & 0.01 \\
\hline Xyris sp.3 & 1860 & $\mathrm{He}$ & 0 & 0 & 0 & 0.08 & 0.08 & 0.09 \\
\hline Xyris sp.4 & 2087 & $\mathrm{He}$ & 0 & 0 & 0 & 0 & 0.02 & 0.01 \\
\hline Xyris sp.5 & 1951 & $\mathrm{He}$ & 0 & 0 & 0 & 0 & 0.04 & 0.01 \\
\hline
\end{tabular}

Voucher: Collect number of MCTB Messias; LF = Life-form; $\mathrm{Ph}=$ phanerophyte; $\mathrm{He}=$ hemicryptophyte; $\mathrm{Cr}=$ cryptophyte; Ch = chamaephyte; $\mathrm{Th}=$ therophyte; ISG = Grasslands in sloped areas, with bare itabirite rocks, near the mountain summit; IPG = Grasslands on the itabirite plateaus, in the middle of the slope, with no more than a $10 \%$ inclination; IWS = Woody savannas in the lower and/or concave parts of the slopes, in valleys or depressions of the itabirite areas; QSG = Grasslands in sloped areas, with bare quartzite rocks, near the mountain summit; QPG = Grasslands on the quartzite plateaus, in the middle of the slope, with no more than a $10 \%$ inclination; QWS = Woody savannas in the lower and concave parts of the slopes, in valleys or depressions of the quartzite areas. 
Table 2. Comparison among the life-form spectra of quartzite and itabirite rocky outcrop sites.

\begin{tabular}{lrrr}
\hline \multicolumn{1}{c}{ Spectra } & DF & $\boldsymbol{X}^{\mathbf{2}}$ & P \\
\hline Floristic vs. frequency & 4 & 7.63 & $>0.106$ \\
Floristic vs. vegetational & 4 & 271.23 & $<0.001$ \\
Frequency vs. vegetational & 4 & 413.8 & $<0.001$ \\
Floristic spectra among the different habitats & 20 & 23.07 & $>0.285$ \\
Frequency spectra among the different habitats & 20 & 98.74 & $<0.001$ \\
Vegetational spectra among the different habitats & 20 & 152.46 & $<0.001$ \\
\hline
\end{tabular}

$\mathrm{DF}=$ Degrees of freedom, $X^{2}=$ Chi-square value.

areas showed a significant difference in their vegetational spectra $\left(X^{2}=80.3\right.$, d.f. $\left.=4, \mathrm{p}<0.001\right)$. The dominance of phanerophytes was greater and the hemicryptophytes was lesser in the itabirite habitats. The woody savannas, in both lithologies, have a greater dominance of phanerophytes and a lesser dominance of hemicryptophytes when compared with the grasslands. The vegetational spectra for both kinds of grasslands in the itabirite areas (ISG, IPG) differed from that of the woody savannas in the same lithology (IWS). The grasslands on the quartzitic plateaus (QPG) were different from QWS by the same pattern. However, the vegetational spectrum in QSG did not show the same standard and isn't statistically different from QWS. The greatest hemicryptophyte dominance among all the studied habitats was found at QPG. Grasslands with bare rocks on itabirite (ISG) differed significantly $(\mathrm{p}<0.05)$ from the plateaus with the same lithology (IPG), mainly due to the greater proportion of hemicryptophytes. ISG also showed a significant difference from QSG, explained by a greater phanerophyte dominance, followed by a lesser dominance of hemicryptophytes and chamaephytes in ISG. The plateaus with canga (IPG) showed the least hemicryptophyte dominance of all the grasslands.

By analyzing all the studied habitats together, the floristic and frequency spectra did not differ significantly from each other $\left(\mathrm{X}^{2}=7.63, \mathrm{p}>0.106\right)$ and both were different from the vegetational one $\left(X^{2}=271.23, \mathrm{p}<0.001\right.$ and $X^{2}=413.8, \mathrm{p}<0.001$ respectively) (Table 2$)$.

Phanerophytes are usually Magnoliopsida, except for Vellozia compacta and Barbacenia flava, while the hemicryptophytes are mainly Liliopsida, from the Poaceae family (Table 1).

\section{Discussion}

The most counted life-forms (phanerophyte and hemicryptophyte) were the same in all the biological spectra but with different patterns of variation. Phanerophytes, chamaephytes and hemicryptophytes are generally well represented in the biological spectra of Brazilian rocky outcrops: ferruginous (Jacobi et al. 2007, Jacobi \& Carmo 2008, Mourão \& Stehmann 2007); quartzitic (Alves \& Kolbek 2009, Conceição \& Giulietti 2002, Conceição \& Pirani 2005, Conceição et al. 2007a); granitic (Caiafa \& Silva 2005); and nepheline-syenite (Ribeiro et al. 2007). A small proportion of therophytes was also found in the biological spectra of other rocky outcrop sites (Conceição \& Pirani 2005, Jacobi et al. 2007, Meirelles et al. 1999, Ribeiro \& Medina 2002, Ribeiro et al. 2007). Bazzaz \& Morse (1991) related that therophytes are expected to become dominant where growing conditions are so adverse that the probability of survival until the second year becomes very small. However, unfavorable growing conditions for part of the year do not hinder the occurrence of perennials, as they favor selection by other strategies, such as dormant structures that enable species to survive in harsh conditions. Therophytes usually dominate in some of the driest and coldest environments of the world (Harrison et al. 2010). Environmental constraints in rocky outcrops might not have been extreme enough to favor the strategy of the therophytes. Ribeiro
\& Medina (2002) suggested that the lack of therophytes in rocky outcrops is due to the difficulty they have to complete their cycle in this harsh environment within the favorable season. According to Ribeiro et al. (2007), therophytes may be rare due to a combination of short growing seasons and low nutrient budgets on rock surfaces that preclude rapid establishment and growth. This is reinforced by the observation that after an extensive wildfire in a rocky outcrop site in Rio de Janeiro, many therophytes established and flowered in large numbers (Ribeiro 2002). According to this author, this was probably due to an input of usually limiting nutrients, mainly phosphorous, that triggered the germination, establishment and growth of annual species.

Some quartzite-sandstone rocky outcrops in Chapada Diamantina showed a higher proportion of chamaephytes than phanerophytes (Conceição \& Giulietti 2002, Conceição et al. 2007a). However, the authors used the Raunkiaer system adapted by Mueller-Dombois \& Ellenberg (1974), where the height separating chamaephytes and phanerophytes is $25 \mathrm{~cm}$ higher. In addition, the species height depends on the environmental conditions. One of the kinds of chamaephytes is the woody, erect subshrubs, with all the characteristics of phanerophytes, except size (Adamson 1939). Some species are facultative shrubs, that is, they are trees under more favorable conditions (Cain 1950). Porto \& Silva (1989) pointed out that the high level of some toxic metals might cause dwarfness. It may explain how a species like Tibouchina heteromalla, usually present in campos rupestres surveys, varies from $0.3 \mathrm{~m}$ to almost $2 \mathrm{~m}$ tall.

The hemicryptophytes are mainly represented by grasses (e.g. Apochloa poliophylla, Axonopus siccus, A. laxiflorus, Echinolaena inflexa and Schizachyrium tenerum), sedges (e.g. Lagenocarpus rigidus and Rhynchospora spp.), Orchidaceae and Bromeliaceae species. The grasses and sedges occur in pools of soil, surrounded by bare rocks, or in the sandy, sharp soil in the quartzitic plateaus or valleys. Orchidaceae and Bromeliaceae are mainly epilythic species showing vegetative reproduction. Some species considered hemicryptophytes in this work e.g. Epidendrum secundum and Coppensia blanchetii were considered chamaephytes by Conceição \& Giulietti (2002). Even though species can appear with different life-forms in different environments, Allan (1937) emphasized the lack of a sharp boundary between chamaephytes and hemicryptophytes and Hagerup (1930) discussed the difficulty of categorizing many short-lived perennials. The environmental constraints resulting from drought and water logging might explain the high dominance of hemicryptophytes in rocky outcrops. They are mainly from Liliopsida, having a fasciculate root system, occupying the superficial layer of the soil. Because the upper layer of the soil contains more organic matter, during the dry season, it has a higher water holding capacity, while on the other hand, during the rainy season, it has a greater oxygen level than the lower ones. It may explain the fact that in the quartzitic plateaus, with poor drainage, hemicryptophytes are more dominant. Besides this, there are other anatomic adaptations favoring monocots in wetlands, as described by Visser et al. (2000). 
The floristic spectrum shows more about richness in each lifeform than the vegetational feature of each environment. There was not any significant difference in the floristic spectra of the different studied habitats, where all the species had the same mean. However, the biological spectra exhibited differences when the species were weighted by a measure of frequency or coverage. It shows that the usefulness of biological spectra is enhanced by adding quantitative data concerning the species. The floristic and frequency spectra were not significantly different, but both differed statistically from the vegetational one. These results evidenced that the frequency evaluation did not add any significant value to the species list used to construct the biological spectra. Raunkiaer (1934) suggested frequency as a descriptor because of the difficulty of counting the individuals of some species. Even though frequency is a measure of abundance, it is influenced by the spatial distribution of individuals (Mueller-Dombois \& Ellenberg 1974) and its relationship with density is logarithmic (Greig-Smith 1983). Frequency is an accurate estimation of density only if there is a random spatial distribution of the individuals, which is not common for plant species, being biased for rare or very common species or for those with clumped distribution (Greig-Smith 1983). According to this author, the advantage of frequency assessment is simply the facility and quickness with which it is obtained. Since density is difficult to obtain in field work for herbaceous communities, some authors have used coverage (Conceição \& Giulietti 2002, Conceição \& Pirani 2005, 2007, Conceição et al. 2007a, b, Wikum \& Shanholtzer 1978). According to Cain (1950), coverage data seems perhaps the most useful, since it is more independent of sample-plot size than are density and frequency. Another advantage of cover as a quantitative measure is that nearly all plant life-forms, from trees to mosses, can be evaluated by the same parameter and thereby in comparable terms (Mueller-Dombois \& Ellenberg 1974). Moreover, this evaluation requires only one third to one fifth of the time used for field work (Wikum \& Shanholtzer 1978), compared with density methods.

Thus, the vegetational spectrum, where the species were weighted by coverage, was quite distinct from the floristic one and provided a more accurate description of the vegetational physiognomy. The proportion of phanerophytes and hemicryptophytes was greater than the other life-forms, which were very reduced when weighted by their dominance. Since the individual biomass is greater for the phanerophyte species, they were more important in the vegetational spectrum. The hemicryptophytes, which usually have vegetative reproduction, showed more individuals forming dense mats. So, the most important contribution to the coverage is given by density. The greater dominance of phanerophytes in itabirite may be explained by the higher occurrence of narrow vertical fissures in the rocks that permit the rooting of taller species and also by the higher level of some nutrients (Vincent \& Meguro 2008). In addition, the higher dominance of Vellozia compacta (a phanerophyte which showed the highest coverage among all the species) in ferruginous campos rupestres, may be associated with tolerance to high levels of heavy metals and drought (Jacobi et al. 2007). These conditions are usually present in the sharp-rock soils as described by Conceição et al. (2007a), Jacobi et al. (2007), Meguro et al. (1977), Meirelles et al. (1997) and Owoseye \& Sanford (1972) and are still harsher in ferruginous soils. This species only grows among rock outcrops, which occur in higher proportion in ferruginous campos rupestres, and is absent in quartzite plateaus where bare rocks are rare. Alves \& Kolbek (2010) related that mechanically unstable sandy soils, like those found in the quartzitic plateaus, can be unavailable for the longevous, shrubby species of Vellozia.

Quartzitic and ferruginous rocky outcrops have similar floristic but quite different vegetational spectra. The greatest difference between the two lithologies was found in the plateau habitats. The greatest dominance of hemicryptophytes covering the sharp, sandy soil was found in the quartzitic plateaus, while in the itabirite plateaus (IPG), the spectrum presented the smallest occurrence of hemicryptophytes among the grasslands. Viana \& Lombardi (2007) also mentioned a lesser occurrence of Poaceae in this kind of environment compared to other kinds of ferruginous rocky outcrops. Phanerophytic shrubs with sclerophyll leaves, common in areas with outcrops like IPG, can extract water from dry soils at low water potential and are typically long-lived. Their high construction costs are amortized over a number of years, but their photosynthetic capacity is low because of internal shading within the leaves and/or high mesophyll resistance to $\mathrm{CO}_{2}$ diffusion (Lloyd et al. 1992). This feature means that sclerophylls can dominate in some dry environments, such as the grasslands with outcrops, but do not compete effectively in wetter ones like the QPG.

The woody savannas, in both lithologies, have a greater dominance of phanerophytes and lesser dominance of hemicryptophytes when compared with the grasslands, which is probably due to the thicker soil that favors the establishment of shrubs or small trees. On the other hand, the lesser dominance of hemicryptophytes may be explained by the high competitiveness of the phanerophyte species. In itabirite areas, both kinds of grassland presented differences when compared to the woody savannas (IWS). The vegetational spectrum in QSG did not show the same pattern and is not statistically different from QWS. However, the most dominant phanerophytes in grasslands are nanophanerophytes, mainly represented by Vellozia compacta, while in QWS, more microphanerophytes were found, varying from 0.7 to $3 \mathrm{~m}$, with shrubs of different specimens of Myrtaceae, Asteraceae, Euphorbiaceae, Lauraceae, Annonaceae, Siparunaceae and others.

The vegetational spectrum would be applied when the aim of the research is the ecological knowledge of the vegetation, rather than the flora of a given site. Cain (1950) suggested that some measure of the relative dominance of each species in the community would provide the most significant data. The vegetational spectra found in these sites fit between phanerophytic and hemicryptophyte. According to Raunkiaer (1934), a phanerophytic climate is typical of the warm humid tropics, while the hemicryptophytic phytoclimate corresponds to a cold-humid climate, typical of high latitudes or high altitudes. The applicability of Raunkiaer's system for tropical communities has been criticized by Sarmiento \& Monasterio (1983) because its assumption that the constraint on plant growth is low winter temperatures, which is clearly not a significant issue in these communities. Batalha \& Martins (2002) discussed the value of the phytoclimate concept when applied to cerrado vegetation, suggesting that water stress, water logging, fire, oligotrophism or aluminum toxicity can be similar to low winter temperatures. Thus, the use of Raunkiaer's system is not only possible, but recommended, when investigating factors, other than climate, that shape the vegetational physiognomy.

The biological spectra of the Brazilian rock outcrop vegetation seem to be somewhat consistent, with phanerophytes and/or chamaephytes and hemicryptophytes always being the most represented classes and with a lack of therophytes. The rather distinct individual differences can be also correlated, in very general terms, with the characteristics of the flora in surrounding areas (Adamson 1939); being the southern portion of Espinhaço Range influenced by the Cerrado and the northern one by the Caatinga (Viana \& Filgueiras 2008). These differences can also be due to the different life-form classification systems used or the plasticity of the height of most species.

Although limited to a small rocky outcrop site, this study did not corroborate Raunkiaer's statement that the frequency spectrum is a good assessment of life-form distribution in plant communities. In addition, the frequency spectrum was not significantly different 
from the floristic one. Thus, for these reasons the frequency spectrum is not suggested in studies of rocky outcrop sites. Similarly to that described by Batalha \& Martins (2004) for cerrado sites, the floristic spectrum is suggested, if working in sufficiently large areas because it could provide an indication of the prevailing phytoclimate. On the other hand, the vegetational spectrum is recommended for small-area analysis, because it provides a detailed description of the physiognomy related with ecological factors. This data analysis supports the suitability of the use of coverage to solve the difficulty of counting individuals in order to construct the vegetational spectra in rocky outcrop sites.

Since the vegetational spectra are different in the different ferruginous rocky outcrop habitats this feature must be considered in the rehabilitation of mining areas. One of the most recent reclamation practices is a topographic reconstruction of mined-out pits, using overburden and spoil material (Griffith \& Toy 2001). Even though this improves the aesthetic view, it could inhibit the reestablishment of the microhabitats described by Jacobi et al. (2007) in ferruginous rocky outcrops. In addition, revegetation projects using a "green carpet" approach, mainly with fast-growing grasses, could give rise to changes in the structure of the vegetation, through hemicryptophyte competitiveness, inhibiting the reestablishment of the natural proportion of life-form groups. Within long-term reclamation project, it is important to consider the life-form proportions as well as the successional dynamics of the community in order to have an efficient restoration of the environmental services of these areas.

\section{Conclusion}

Not only lithology but other aspects of the environment, like geomorphology, influence the distribution of plant life-forms in rocky outcrop habitats. This makes the definition "High Altitude Rupestrian Complex" (Semir 1991, Benites et al. 2003) more appropriate as a phytogeographic description of the rocky outcrop of the Espinhaço Range. Phanerophytes and hemicryptophytes were the life-forms with a higher coverage in the studied areas. The ferruginous campos rupestres have higher phanerophyte and lower hemicryptophyte coverages than the quartzite ones. The lower and/or concave parts of the slopes have a greater phanerophyte and a lesser hemicryptophyte dominance than the two other geomorphologic habitats with grasslands. The floristic and frequency spectra did not differ from each other, but both differed from the vegetational one. The vegetational spectrum was more efficient for the studied rocky outcrop comparison. This study will support the development of restoration projects of campos rupestres by improving the knowledge of flora composition, structure and function.

\section{Acknowledgments}

We would like to thank to SAMARCO S.A. for permitting this research, FAPEMIG, for the financial support CRA - APQ00601-08, Jorge L. Silva, Auria Tonaco and Eduardo Ataíde for field assistance and also the taxonomists for species identifications: C. Kameyama (Acanthaceae); A. Lobão (Annonaceae); A. Rapini (Apocynaceae); M. Nadruz (Araceae); A.M. Teles (Asteraceae); R.S. Araujo (Bignoniaceae); R. Forzza (Bromeliaceae); M. Kirizawa (Dioscoreaceae), M. Trovó and P. Sano (Eriocaulaceae); M.I.B. Loyolla (Erythroxylaceae); I. Cordeiro (Euphorbiaceae, Phyllanthaceae and Gentianaceae); A.O. Araujo (Gesneriaceae); A. Quinet (Lauraceae); P.C.B. Souza (Lentibulariaceae); E. Miranda (Loganiaceae); V.F. Dutra (Leguminosae); C.H.R. Paula (Loranthaceae); T.B. Cavalcanti (Lythraceae); M. Bovini (Malvaceae); R. Romero and J. Baungratz (Melastomataceae); M.F. Freitas (Myrsinaceae); M. Sobral (Myrtaceae). C. Van den Berg and
J.A.N. Batista (Orchidaceae); L. Bernacci (Passifloraceae); M.S. Marchioretto (Phytollacaceae); A.L. Peixoto (Siparunaceae); J.F. Pastore (Polygalaceae); W. Marcondes-Ferreira (Polygonaceae); P.L. Viana (Poaceae); L.C.N. Melo and J.E. Casarino (Pteridophyta); M.S. Ferrucci (Sapindaceae); J.R. Stehmann (Solanaceae); R. Andreata (Smilacaceae); F.O.Souza-Buturi (Styracaceae), M.F. Salimena (Verbenaceae); R. Mello-Silva (Velloziaceae).

\section{References}

ADAMSON, R.S. 1939. The classification of life-forms of plants. Bot. Rev. 5(10):546-561. http://dx.doi.org/10.1007/BF02868932

ALLAN, H.H. 1937. A consideration of the "biological spectra" of New Zealand. J. Ecol. 25(1):116-152. http://dx.doi.org/10.2307/2256338

ALVES, R.J.V. \& KOLBEK, J. 2009. Summit vascular flora of Serra de São José, Minas Gerais, Brazil. Check List 5(1):35-73.

ALVES, R.J.V. \& KOLBEK, J. 2010. Vegetation strategy of Vellozia crinita (Velloziaceae). Biologia 65(2):254-264. http://dx.doi.org/10.2478/ s11756-010-0005-y

ANGIOSPERM PHYLOGENY GROUP - APG. 2009. An update of the Angiosperm Phylogeny Group classification for the orders and families of flowering plants: APG III. Bot. J. Linn. Soc.161(2):105-121. http:// dx.doi.org/10.1111/j.1095-8339.2009.00996.x

BATALHA, M.A. \& MARTINS, F.R. 2002. Biological spectra of cerrado sites. Flora 197(6):452-460.

BATALHA, M.A. \& MARTINS, F.R. 2004. Floristic, frequency and vegetation life-form spectra of a cerrado site. Braz. J. Biol. 64(2):203-209. http://dx.doi.org/10.1590/S1519-69842004000200004

BAZZAZ, F.A. \& MORSE, S.R. 1991. Annual plants: potential responses to multiple stresses. In Response of plants to multiple stresses (H.A. Mooney, W.E. Winner \& E.J. Pel, eds.). Academic Press, London, p. 283-305.

BEGON, M., HARPER, J.L. \& TOWNSEND, C.R. 1996. Ecology: individuals, populations and communities. $3^{\text {nd }}$ ed. Blackwell, Oxford.

BENITES, V.M., CAIAFA, A.N., MENDONÇA, E.S., SCHAEFER, C.E. \& KER, J.C. 2003. Solos e vegetação nos complexos rupestres de altitude da Mantiqueira e do Espinhaço. Floresta Ambient. 10(1):76-85.

BENITES, V.M., SCHAEFER, C.E.G.R., SIMAS, F.N.B. \& SANTOS, H.G. 2007. Soil associated with rock outcrops in the Brazilian mountain ranges Mantiqueira and Espinhaço. Rev. Bras. Bot. 30(4):569-577. http://dx.doi. org/10.1590/S0100-84042007000400003

CAIAFA, N.A. \& SILVA, A.F. 2005. Composição florística e espectro biológico de um campo de altitude no Parque Estadual da Serra do Brigadeiro, Minas Gerais - Brasil. Rodriguesia 56(87):163-173.

CAIN, S.A. 1950. Life-forms and phytoclimate. Bot. Rev. 16(1):1-32. http:// dx.doi.org/10.1007/BF02879783

CONCEIÇÃO, A.A. \& GIULIETTI, A.M. 2002. Composição florística e aspectos estruturais de campo rupestre em dois platôs do Morro do Pai Inácio, Chapada Diamantina, Bahia, Brasil. Hoehnea 29(1):37-48.

CONCEIÇÃO, A.A., GIULIETTI, A.M. \& MEIRELLES, S.T. 2007a. Ilhas de vegetação em afloramentos de quartzito-arenito no Morro do Pai Inácio, Chapada Diamantina, Bahia, Brasil. Acta Bot. Bras. 21(2):335-347.

CONCEIÇÃO, A.A. \& PIRANI, J.R. 2005. Delimitação de habitats em campos rupestres na Chapada Diamantina, Bahia: substratos, composição florística e aspectos estruturais. Bol. Bot. 23(1):85-111.

CONCEIÇÃO, A.A. \& PIRANI, J.R. 2007. Diversidade em quatro áreas de campos rupestres na Chapada Diamantina, Bahia, Brasil: espécies distintas, mas riquezas similares. Rodriguesia 58(1):193-206.

CONCEIÇÃO, A.A, PIRANI, J.R. \& MEIRELLES, S.T. 2007b. Floristics, structure and soil of insular vegetation in four quartzite-sandstone outcrops of "Chapada Diamantina", Northeast Brazil. Rev. Bras. Bot. 30(4):641-656.

GAFF, D.F. 1977. Desiccation tolerance vascular plants of Southern Africa. Oecologia 31(1):95-109. http://dx.doi.org/10.1007/BF00348713 
GAFF, D.F. 1987. Desiccation tolerant plants in South America. Oecologia 74(1):133-136. http://dx.doi.org/10.1007/BF00377357

GIULIETTI, A.M. \& PIRANI, J.R. 1988. Patterns of geographic distribution of some plant species from the Espinhaço Range, Minas Gerais and Bahia, Brazil. In Proceedings of a workshop on Neotropical Distribution Patterns (P.E. Vanzolini \& W.R. Heyer, eds.). Academia Brasileira de Ciências, Rio de Janeiro, p.39-69.

GREIG-SMITH, P. 1983. Quantitative plant ecology. $3^{\text {nd }}$ ed. Blackwell, Oxford.

GRIFFITH, J. \& TOY, T.J. 2001. Evolution in revegetation of iron-ore mines in Minas Gerais State, Brazil. Unasylva 52(1):9-15.

HAGERUP, O. 1930. Étude des types biologiques de Raunkiaer dans la flore autour de Tombouctou. K. Dan. Vidensk. Selsk. Skr. 9(4):1-116.

HARLEY, R.M. \& SIMMONS, N.A. 1986. Florula of Mucugê, Chapada Diamantina, Bahia, Brazil. Royal Botanic Gardens, Kew.

HARRISON, S.P., PRENTICE, I.C., BARBONI, D., KOHFELD, K.E., NI, J. \& SUTRA, J.P. 2010. Ecophysiological and bioclimatic foundations for a global plant functional classification. J. Veg. Sci. 21(2):300-317. http://dx.doi.org/10.1111/j.1654-1103.2009.01144.x

JACOBI, C.M. \& CARMO, F.F. 2008. Diversidade dos campos rupestres ferruginosos no Quadrilátero Ferrífero, MG. Megadiversidade 4(1-2):24-32.

JACOBI, C.M., CARMO, F.F., VINCENT, R.C. \& STEHMANN, J.R. 2007. Plant communities on ironstone outcrops - a diverse and endangered Brazilian ecosystem. Biodivers. Conserv. 16(7):2185-2200. http://dx.doi. org/10.1007/s10531-007-9156-8

LOMOLINO, M.V., RIDDLE, B.R. \& BROWN, J.H. 2005. Biogeography. 3 ed. Sinauer Associate Inc., Sunderland.

LOYD, J., SYVERTSEN, J.P., KRIEDEMANN, P.E. \& FARQUHAR, G.D. 1992. Low conductances for CO2 diffusion from stomata to the sites of carboxylation in leaves of woody species. Plant Cell Environ. 15(8):873-899. http://dx.doi.org/10.1111/j.1365-3040.1992.tb01021.x

MEGURO, M., JOLY, C.A. \& BITTENCOURT, M.M. 1977. Stress hídrico e alguns aspectos do comportamento fisiológico em Xerophyta plicata Spreng. Velloziaceae. Bol. Bot. 5(1):27-42.

MEGURO, M., PIRANI, J.R., GIULIETTI, A.M. \& MELLO-SILVA, R. 1994. Phytophysiognomy and composition of the vegetation of Serra do Ambrósio, Minas Gerais, Brazil. Rev. Bras. Bot. 17(2):149-166.

MEIRELLES, S.T., MATTOS, E.A. \& SILVA, A.C. 1997. Potential desiccation tolerant vascular plants from Southeastern Brazil. Pol. J. Environ. Stud. 6(4):17-21.

MEIRELLES, S.T., PIVELLO, V.R. \& JOLY, C.A. 1999. The vegetation of granite rock outcrops in Rio de Janeiro, Brazil, and the need for its protection. Environ. Conserv. 26(1):10-20. http://dx.doi.org/10.1017/ S0376892999000041

MERA, A.G., HAGEN, M.A. \& ORELLANA, J.A.V. 1999. Aerophyte, a new life form in Raunkiaer's classification? J. Veg. Sci. 10(1):65-68.

MOURÃO, A. \& STEHMANN, J.R. 2007. Levantamento da flora do campo rupestre sobre canga hematítica couraçada remanescente na mina do Brucutu, Barão de Cocais, Minas Gerais, Brasil. Rodriguesia 58(4):775-786.

MUELLER-DOMBOIS, D. \& ELLENBERG, H. 1974. Aims and methods of vegetation ecology. $2^{\text {nd }}$ ed. John Willey $\&$ Sons, New York.

NIMER, E. 1989. Climatologia do Brasil. $2^{\text {nd }}$ ed. Instituto Brasileiro de Geografia e Estatística, Rio de Janeiro.

OWOSEYE, J.A. \& SANFORD, W.W. 1972. An ecological study of Vellozia schnitzleinia, a drought-enduring plant of northern Nigeria. J. Ecol. 60(3):807-817. http://dx.doi.org/10.2307/2258567

POREMBSKI, S. \& BARTHLOTT, W. 2000. Granitic and gneissic outcrops (inselbergs) as centers of diversity for desiccation-tolerant vascular plants. Plant Ecol. 151(1):19-28. http://dx.doi.org/10.1023/A:1026565817218
POREMBSKI, S., BARTHLOTT, W., DÖRRSTOCK, S. \& BIEDINGER, N. 1994 Vegetation of rock outcrops in Guinea: granite inselbergs, sandstone table mountains and ferricretes - remarks on species numbers and endemism. Flora 189(4):315-326.

POREMBSKI, S., MARTINELLI, G., OHLEMÜLLER, R. \& BARTHLOTT, W. 1998. Diversity and ecology of saxicolous vegetation mats on inselbergs in the Brazilian Atlantic rainforest. Divers. Distrib. 4(3):107-119. http://dx.doi.org/10.1046/j.1365-2699.1998.00013.x

PORTO, M.L. \& SILVA, M.F.F. 1989. Tipos de vegetação metalófila em áreas da Serra de Carajás e de Minas Gerais. Acta Bot. Bras. 3(1):13-21.

QUEIROZ, L.P., SENA, T.S.N. \& COSTA, M.J.S.L. 1996. Flora vascular da Serra da Jibóia, Santa Terezinha, Bahia. I: O campo rupestre. Sitientibus 15(1):27-40.

RAUNKIAER, C. 1934. The life forms of plants and statistical geography. Claredon Press, Oxford.

RIBEIRO, K.T. 2002. Estrutura, dinâmica e funcionalidade em comunidades rupícolas no Planalto do Itatiaia. Tese de doutorado, Universidade Federal do Rio de Janeiro, Rio de Janeiro.

RIBEIRO, K.T. \& MEDINA, B.M.O. 2002. Estrutura, dinâmica e biogeografia das ilhas de vegetação sobre rocha do Planalto do Itatiaia, RJ. Bol. Parq. Nac. Itatiaia 10(1):1-84.

RIBEIRO, K.T., MEDINA, B.M.O. \& SCARANO, F.R. 2007. Composição de espécies e relações biogeográficas da flora sobre afloramentos rochosos no Planalto do Itatiaia, SE do Brasil. Rev. Bras. Bot. 30(4):623-639. http:// dx.doi.org/10.1590/S0100-84042007000400008

ROSIÈRE, C.A. \& CHEMALE JUNIOR, F. 2000. Itabiritos e minérios de ferro de alto teor do Quadrilátero ferrífero - uma visão geral e discussão. Geonomos 8(2):27-43.

SARMIENTO, G. \& MONASTERIO, M. 1983. Life forms and phenology. In Ecosystems of the world: tropical savannas (F. Bourliére, ed.). Elsevier, Amsterdam, p. 79-108.

SEMIR, J. 1991. Revisão taxonômica de Lychnophora Mart. (Vernoniaeae: Compositae). Tese de doutorado, Universidade Estadual de Campinas, Campinas.

SILVA, M.F.F. 1991. Análise florística da vegetação que cresce sobre canga hematítica em Carajás - Pará (Brasil). Bol. Mus. Para. Emilio Goeldi, Bot. 7(1):79-105.

VIANA, P.L. \& FILGUEIRAS, T.S. 2008. Inventário e distribuição geográfica das gramíneas (Poaceae) na Cadeia do Espinhaço, Brasil. Megadiversidade 4(1-2):99-116.

VIANA, P.L. \& LOMBARDI, J.A. 2007. Florística e caracterização dos campos rupestres sobre canga na Serra da Calçada, Minas Gerais, Brasil. Rodriguesia 58(1):159-177.

VINCENT, R.C. 2004. Florística, fitossociologia e relações entre a vegetação e o solo em áreas de campos ferruginosos no Quadrilátero Ferrífero, Minas Gerais. Tese de doutorado, Universidade de São Paulo, São Paulo.

VINCENT, R.C. \& MEGURO, M.M. 2008. Influence of soil properties on the abundance of plants species in ferruginous rocky soils vegetation, southeastern Brazil. Rev. Bras. Bot. 31(3):377-388. http://dx.doi. org/10.1590/S0100-84042008000300002

VISSER, E.J.W., COLMER, T.D., BLOM, C.W.P.M. \& VOESENEK, C.J. 2000. Changes in growth, porosity and radical oxygen loss from adventitious roots of selected mono- and dicotyledonous wetland species with contrasting types of aerenchyma. Plant Cell Environ. 23(11):1237-1245. http://dx.doi.org/10.1046/j.1365-3040.2000.00628.x

WESTHOFF, V. \& MAAREL, E. 1978. The Braun-Blanquet approach. In Classification of plant communities (R.H. Whittaker, ed.). Dr. W. Junk, Netherlands, p. 289-374.

WIKUM, D. \& SHANHOLTZER, G. 1978. Application of the Braun-Blanquet cover-abundance scale for vegetation analysis in land development studies. Environ. Manage. 2(4):323-329. http://dx.doi.org/10.1007/ BF01866672

ZAR, J.H. 1999. Biostatistical analysis. $4^{\text {nd }}$ ed. Prentice Hall, New Jersey. 